\title{
Optimal Conditionally Unbiased Bounded-Influence Inference in Dynamic Location and Scale Models*
}

\author{
Loriano Mancini $^{a}$, Elvezio Ronchetti ${ }^{a, b}$ and Fabio Trojani ${ }^{a}$ \\ ${ }^{a}$ Institute of Finance, University of Lugano, Switzerland \\ ${ }^{b}$ Dept. of Econometrics, University of Geneva, Switzerland
}

First Version: January 2003

Revised: November 2003

Keywords: Time series models, $M$-estimators, influence function, robust estimation and testing.

JEL Classification: C12, C13, C14, C22.

\footnotetext{
${ }^{*}$ Correspondence Information: Loriano Mancini, Institute of Finance, University of Lugano, Via Buffi 13, CH-6900 Lugano, tel: +41 (0)9191247 86. loriano.mancini@lu.unisi.ch. E-mail addresses for Elvezio Ronchetti and Fabio Trojani: elvezio.ronchetti@metri.unige.ch, fabio.trojani@lu.unisi.ch. Loriano Mancini and Fabio Trojani gratefully acknowledge the financial support of the Swiss National Science Foundation (grant 12-65196.01 and NCCR FINRISK). The authors thank Giovanni Barone-Adesi, Claudio Ortelli, Claudia Ravanelli, the referees, the associate editor, and the editor for their valuable suggestions.
} 


\begin{abstract}
This paper studies the local robustness of estimators and tests for the conditional location and scale parameters in a strictly stationary time series model. We first derive optimal bounded-influence estimators for such settings under a conditionally Gaussian reference model. Based on these results, optimal bounded-influence versions of the classical likelihood-based tests for parametric hypotheses are obtained. We propose a feasible and efficient algorithm for the computation of our robust estimators, which makes use of analytical Laplace approximations to estimate the auxiliary recentering vectors ensuring Fisher consistency in robust estimation. This strongly reduces the necessary computation time by avoiding the simulation of multidimensional integrals, a task that has typically to be addressed in the robust estimation of nonlinear models for time series. In some Monte Carlo simulations of an $\mathrm{AR}(1)-\mathrm{ARCH}(1)$ process we show that our robust statistics maintain a very high efficiency under ideal model conditions and at the same time perform very satisfactorily under several forms of departure from conditional normality. On the contrary, classical Pseudo Maximum Likelihood inference procedures are found to be strongly biased and highly inefficient under such local model misspecifications. These patterns are confirmed by an application to robust testing for ARCH.
\end{abstract}




\section{Introduction}

This paper studies the local robustness properties of estimation and testing procedures for the conditional location and scale parameters of a strictly stationary time series model.

The class of conditional location and scale time series models is quite broad and includes several well-known dynamic models largely applied in economics and empirical finance, such as pure conditional scale models (like ARCH models; Engle (1982)) or models that jointly parameterize the conditional location and the scale of the given time series (like for instance ARCH in mean models; Engle, Lilien and Robins (1987)). Typically, classical (non robust) estimation of the parameters of such models is obtained by means of a Pseudo Maximum Likelihood (PML) approach based on the nominal assumption of a conditionally Gaussian log-likelihood; see also Bollerslev and Wooldridge (1992). Such PML estimators are based on an unbounded conditional score function, implying - as shown below - an unbounded time series influence function (IF, Künsch (1984) and Hampel (1974)). As a consequence, PML estimators for conditional location and scale models are not robust under local departures from conditional normality.

In this paper we propose a new class of inference procedures which are robust to local nonparametric misspecifications of a parametric, conditionally Gaussian, location and scale model. More specifically, we consider the class of robust, conditionally unbiased, $M$-estimators for the parameters of conditional location and scale models and derive the optimal (i.e. the most efficient) robust estimator within this class. Based on such estimators, several Maximum Likelihood (ML)-type bounded-influence tests for parametric hypotheses on the parameters of the conditional location and scale equations are then obtained following the general approach in Heritier and Ronchetti (1994) and Ronchetti and Trojani (2001).

The need for robust procedures in estimation and testing has been stressed by many authors and is now widely recognized both in the statistical and the econometric literature; cf. for instance Hampel (1974), Koenker and Bassett (1978), Huber (1981), Koenker (1982), Hampel et al. (1986), Peracchi (1990), and more recently Markatou and Ronchetti (1997), Krishnakumar and Ronchetti (1997), Ronchetti and Trojani (2001), Ortelli and Trojani (2002). However, the problem of the robust estimation for the parameters of conditional location and scale models has been considered so far by very few authors and only from the specific perspective of high breakdown estimation. Even less attention has been devoted 
to robust inference within conditional location and scale models. High breakdown estimators resistant to large amount of contamination have been proposed by Sakata and White (1998) and Muler and Yohai (1999). These estimators are very computationally intensive and cannot be applied to estimate the parameters of a class of broadly applied models - such as for instance threshold ARCH or ARCH in mean models.

This paper derives optimal bounded-influence estimation and testing procedures for a general conditional location and scale model, which are computationally only slightly more demanding than those required by a classical PML estimation of such models. The more specific contributions to the current literature are the following.

First, we characterize the robustness of conditionally unbiased $M$-estimators for nonlinear conditional location and scale models by computing the time series IF of Künsch (1984) for the implied asymptotic functional estimator. This is a first necessary step which allows us to construct robust statistical procedures which can control for $(i)$ the local asymptotic bias on the parameter estimates and (ii) the local asymptotic distortion on the level and the power of ML-type tests.

Second, we derive the optimal bounded-influence estimator for the parameters of conditional location and scale models under a conditionally Gaussian reference model. This extends the optimality result in Künsch (1984) and the application of optimal conditionally unbiased $M$-estimators in Künsch, Stefanski and Carroll (1989) to general nonlinear second order dynamic models. Based on these results, optimal bounded-influence versions of the classical Wald, score and likelihood ratio tests are derived along the general lines proposed in Heritier and Ronchetti (1994) and Ronchetti and Trojani (2001).

Third, we propose a feasible algorithm for the computation of our optimal robust estimators, which can be easily implemented in standard packages, such as Matlab. This procedure is based on a truncating procedure which uses a set of Huber's weights to downweight the impact of influential observations. Fisher consistency at the model is preserved by means of some auxiliary recentering vectors, which in a time series setting have generally to be computed by simulations - as for instance in a Robust Efficient Method of Moments (REMM, Ortelli and Trojani (2002)) setting. Using the conditional unbiasedness of our estimator we provide analytical Laplace approximations for such vectors which strongly reduce the 
necessary computation time by avoiding the simulation of multidimensional integrals.

Fourth, we study by Monte Carlo simulation the efficiency and the robustness properties of our estimator. We estimate a simple $\mathrm{AR}(1)-\mathrm{ARCH}(1)$ process under several models of local contamination of a conditionally Gaussian process. Under the Gaussian reference model the classical ML estimator and our robust estimator have essentially the same efficiency. On the contrary, under local deviations from conditional normality classical PML estimators, tests and confidence intervals are found to be strongly biased and highly inefficient, while robust procedures perform very satisfactorily.

Finally, we present an application to robust testing for ARCH where robust procedures help in identifying ARCH structures which could not be detected using the classical inference approach.

The structure of the paper is as follows. Section 2 introduces conditional location-scale models and the corresponding classical $M$-estimation procedure. Section 3 computes the time series IF for conditionally unbiased $M$-estimators. The asymptotic bias on the parameter estimates induced by local deviations from the conditional Gaussian reference model is then approximated. In a second step, the optimal robust estimator is derived and the optimality of robust inference procedures based on such estimators is discussed. The section is concluded by deriving analytic approximations for the auxiliary recentering vectors in our robust estimation and by presenting an algorithm to compute our robust estimator in applications. Section 5 presents the Monte Carlo experiments where the performance of our robust estimation and inference approach is evaluated in the setting of an $\mathrm{AR}(1)-\mathrm{ARCH}(1)$ model. The empirical application to testing for ARCH is presented in Section 6. Section 7 summarizes and concludes.

\section{Conditionally Unbiased $M$-estimators}

Let $\mathcal{Y}:=\left(y_{t}\right)_{t \in \mathbb{Z}}$ be a real valued strictly stationary random sequence on the probability space $\left(\mathbb{R}^{\infty}, \mathcal{F}, \mathbb{P}_{*}\right)$ and $\mathcal{P}:=\left\{\mathbb{P}_{\theta}, \theta \in \Theta \subseteq \mathbb{R}^{p}\right\}$ be some parametric model for $\mathbb{P}_{*}$. Under any model $\mathbb{P}_{\theta_{0}}$, the random variable $y_{t}$ has a conditionally Gaussian distribution, $y_{t} \mid \mathcal{F}_{t-1} \sim \mathcal{N}\left(\mu_{t}\left(\theta_{0}\right), \sigma_{t}^{2}\left(\theta_{0}\right)\right)$. Specifically,

$$
\begin{aligned}
y_{t} & =\mu_{t}\left(\theta_{0}\right)+\varepsilon_{t}\left(\theta_{0}\right), \\
\varepsilon_{t}^{2}\left(\theta_{0}\right) & =\sigma_{t}^{2}\left(\theta_{0}\right)+\nu_{t}\left(\theta_{0}\right),
\end{aligned}
$$


where $\mu_{t}\left(\theta_{0}\right)$ and $\sigma_{t}^{2}\left(\theta_{0}\right)$ parameterize the conditional mean and the conditional variance of $y_{t}$ given the information $\mathcal{F}_{t-1}$ up to time $t-1$. Finally, denote by $y_{1}^{m}:=\left(y_{1}, \ldots, y_{m}\right)$ the finite random sequence of $\mathcal{Y}$ and by $\mathbb{P}_{\theta_{0}}^{m}$ the $m$-dimensional marginal distribution of $y_{1}^{m}$ induced by $\mathbb{P}_{\theta_{0}}$. Model (1) covers a broad class of well-known parametric models for time series. A general example is the following.

Example 2.1 Double threshold $\mathrm{AR}(1)-\mathrm{ARCH}(1)$ models with volatility asymmetries (see for instance Li and Li (1996) and Glosten, Jagannathan and Runkle (1993)) assume the specification

$$
\begin{aligned}
& \mu_{t}\left(\theta_{0}\right)=\rho_{0}+\left(\rho_{1}+\rho_{2} d_{1, t-1}\right) y_{t-1} \\
& \sigma_{t}^{2}\left(\theta_{0}\right)=\alpha_{0}+\left(\alpha_{1}+\alpha_{2} d_{2, t-1}\right)\left(y_{t-1}-\rho_{0}-\left(\rho_{1}+\rho_{2} d_{1, t-2}\right) y_{t-2}\right)^{2}+\alpha_{3} d_{1, t-1}
\end{aligned}
$$

with the dummy variable $d_{1, t-1}=1$ if $\rho_{0}+\rho_{1} y_{t-1}>0$ and zero otherwise, $d_{2, t-1}=1$ if $\varepsilon_{t-1}\left(\theta_{0}\right)<0$ and zero otherwise.

Model (1) includes linear autoregressive models as a straightforward special case. Künsch (1984) defined a time series influence function (IF) in this context and derived an optimal bounded-influence estimator for the parameters of an $\operatorname{AR}(p)$ model. Martin and Yohai (1986) provided bounded-influence estimators for AR and MA models and studied the asymptotic bias implied by additive and innovative outliers. ARCH models (cf. Engle (1982)) are also special cases of model (1). Bounded-influence estimators for such models are available in the class of robust GMM (RGMM) or robust EMM (REMM) estimators; cf. Ronchetti and Trojani (2001) and Ortelli and Trojani (2002). Muler and Yohai (1999) considered explicitly the pure (no location) ARCH setting from the perspective of high breakdown estimation. Sakata and White (1998) developed high breakdown estimators for conditional location and scale models that include ARCH models as special cases. However, their high breakdown estimators cannot be applied directly to all models of the form (1) because they assume a partitioned parameter space $\Theta=\Theta_{1} \times \Theta_{2}$ in order to imply $\mu_{t}\left(\theta_{0}\right)=\mu_{t}\left(\theta_{1}\right)$, for $\theta_{1} \in \Theta_{1}$. Moreover, all the above robust estimators are much more computationally intensive than the one proposed in this paper already for simple processes, like for instance ARCH models.

In robust inference, model (1) is interpreted as an "approximate" description of the true data generating process $\mathbb{P}_{*}$. Hence, our aim is to derive efficient and computationally undemanding locally robust procedures for inference on the parameters of model (1) when the data distribution $\mathbb{P}_{*}$ is in some non- 
parametric neighborhood of the reference model $\mathbb{P}_{\theta_{0}}$. To this end we consider the class of conditionally unbiased $M$-estimators for $\theta_{0}$ and a functional $M$-estimator $a(\cdot)$,

$$
a: \operatorname{dom}(a) \subset \mathcal{M}_{\text {stat }}^{m} \longrightarrow \Theta
$$

where $\mathcal{M}_{\text {stat }}^{m}:=\{m$-dimensional marginals of strictly stationary processes $\}$ and $m \in \mathbb{N} \backslash\{\infty\}$. In particular, this excludes GARCH models (Bollerslev (1986)) from our robust analysis.

The estimator $a(\cdot)$ is implicitly defined by an estimating function $\psi: \mathbb{R}^{m} \times \Theta \longrightarrow \mathbb{R}^{p}$ and some conditional moment conditions

$$
E_{\theta_{0}}\left[\psi\left(y_{1}^{m} ; a\left(\mathbb{P}_{\theta_{0}}^{m}\right)\right) \mid \mathcal{F}_{m-1}\right]=0
$$

which hold for a unique $\theta_{0} \in \Theta$. By construction $\left(\psi\left(y_{1+t}^{m+t} ; \theta_{0}\right)\right)_{t \in \mathbb{Z}}$ is a martingale difference sequence under $\mathbb{P}_{\theta_{0}}$. Thus, $a(\cdot)$ is conditionally Fisher consistent and the asymptotic estimating equation for $\theta_{0}$ is

$$
E_{\theta_{0}}\left[\psi\left(y_{1}^{m} ; a\left(\mathbb{P}_{\theta_{0}}^{m}\right)\right)\right]=0 .
$$

For example, the conditionally Gaussian score function $s$

$$
s\left(y_{1}^{m} ; \theta_{0}\right)=-k_{1, m}+k_{2, m} u_{m}\left(\theta_{0}\right)+k_{1, m} u_{m}\left(\theta_{0}\right)^{2},
$$

where $u_{m}\left(\theta_{0}\right)=\varepsilon_{m}\left(\theta_{0}\right) \sigma_{m}\left(\theta_{0}\right)^{-1}$ and

$$
k_{1, m}:=\left.\frac{1}{2 \sigma_{m}^{2}\left(\theta_{0}\right)} \frac{\partial \sigma_{m}^{2}(\theta)}{\partial \theta}\right|_{\theta=\theta_{0}}, \quad k_{2, m}:=\left.\frac{1}{\sigma_{m}\left(\theta_{0}\right)} \frac{\partial \mu_{m}(\theta)}{\partial \theta}\right|_{\theta=\theta_{0}},
$$

defines a conditionally unbiased $M$-estimator of $\theta_{0}$. In this setting, different specifications of $\mu_{m}\left(\theta_{0}\right)$ and $\sigma_{m}^{2}\left(\theta_{0}\right)$ are easily accommodated in the $\mathcal{F}_{m-1}$-measurable random vectors $k_{1, m}$ and $k_{2, m}$.

The estimator $a(\cdot)$ depends on $m$ process coordinates which are entirely determined by the parametric reference model (1). For instance, in Example 2.1 we had $m=3$. The $M$-estimator of $\theta_{0}$ solves the finite sample estimating equations

$$
n^{-1} \sum_{t=1}^{n} \psi\left(\tilde{y}_{t-m+1}^{t} ; \hat{\theta}_{n}\right)=0
$$

which are the finite sample version of the asymptotic condition (4), where $\tilde{y}_{2-m}^{n}$ are sample observations of the process $\mathcal{Y}$. Under model $\mathbb{P}_{\theta_{0}}^{m}, \sqrt{n}\left(\hat{\theta}_{n}-\theta_{0}\right)$ converges in distribution to the Gaussian distribution 
$\mathcal{N}\left(0, V\left(\psi ; \theta_{0}\right)\right)$, where $V(\psi ; \theta):=J(\theta)^{-1} I(\theta) J(\theta)^{-1}$ with

$$
J(\theta):=E_{\theta}\left[-\frac{\partial \psi\left(y_{1}^{m} ; \theta\right)}{\partial \theta^{\top}}\right], \quad I(\theta):=E_{\theta}\left[\psi\left(y_{1}^{m} ; \theta\right) \psi\left(y_{1}^{m} ; \theta\right)^{\top}\right] ;
$$

cf. Bollerslev and Wooldridge (1992), p. 148. In particular, under the reference model $\mathbb{P}_{\theta_{0}}^{m}$, the simple expression for $I(\theta)$ is implied by condition (3).

\section{Locally Robust Estimation}

In this section we allow $\mathbb{P}_{*}$ to be in a nonparametric neighborhood of the reference model $\mathbb{P}_{\theta_{0}}$. In particular, $\mathbb{P}_{\theta_{0}}$ can be dynamically misspecified for $\mathbb{P}_{*}$. As we focus on local robustness we consider local deviations from $\mathbb{P}_{\theta_{0}}^{m}$. Therefore, we assume that $\mathbb{P}_{*}^{m}$ is in the following nonparametric neighborhood $\mathcal{U}^{\eta}\left(\mathbb{P}_{\theta_{0}}^{m}\right)$ of $\mathbb{P}_{\theta_{0}}^{m}$,

$$
\mathcal{U}^{\eta}\left(\mathbb{P}_{\theta_{0}}^{m}\right):=\left\{\mathbb{P}_{\eta}^{m}=(1-\eta) \mathbb{P}_{\theta_{0}}^{m}+\eta \mathbb{G}^{m}, \quad \eta \leq b, b \in[0,1], \mathbb{G}^{m} \in \mathcal{M}_{\text {stat }}^{m}\right\}
$$

The neighborhood defined in (7) is a simple way to formalize local perturbations of the model $\mathbb{P}_{\theta_{0}}^{m}$. Notice that $d_{k}\left(\mathbb{P}_{\eta}^{m}, \mathbb{P}_{\theta_{0}}^{m}\right) \leq \eta$ for all $\mathbb{G}^{m} \in \mathcal{M}_{\text {stat }}^{m}$, where $d_{k}(\cdot, \cdot)$ denotes the Kolmogorov distance.

\subsection{Time Series Influence Function}

Robust procedures aim at the estimation of the parameter $\theta_{0}$ when local deviations from the reference model $\mathbb{P}_{\theta_{0}}^{m}$ are allowed. Such deviations induce an asymptotic bias on the functional estimator $a(\cdot)$, defined by

$$
\text { bias }:=a\left(\mathbb{P}_{*}^{m}\right)-a\left(\mathbb{P}_{\theta_{0}}^{m}\right)=a\left(\mathbb{P}_{*}^{m}\right)-\theta_{0} \text {. }
$$

For a robust inference on $\theta_{0}$, the standard robustness condition on the estimator $a(\cdot)$ is a bounded asymptotic bias. To describe the linearized asymptotic bias of $a(\cdot)$ under some model $\mathbb{P}_{\eta}^{m} \in \mathcal{U}^{\eta}\left(\mathbb{P}_{\theta_{0}}^{m}\right)$ we consider the first order von Mises (1947) expansion of $a(\cdot)$ at $\mathbb{P}_{\theta_{0}}^{m}$ (cf. for instance Fernholz (1983)),

$$
a\left(\mathbb{P}_{\eta}^{m}\right)-a\left(\mathbb{P}_{\theta_{0}}^{m}\right)=\eta a^{\prime}\left(\theta_{0}, \mathbb{G}^{m}\right)+o\left(\left\|\mathbb{P}_{\eta}^{m}-\mathbb{P}_{\theta_{0}}^{m}\right\|\right),
$$

where $a^{\prime}\left(\theta_{0}, \mathbb{G}^{m}\right)$ is the Gâteaux derivative of $a(\cdot)$ in the direction $\mathbb{G}^{m}-\mathbb{P}_{\theta_{0}}^{m}$, defined by

$$
a^{\prime}\left(\theta_{0}, \mathbb{G}^{m}\right):=\lim _{\eta \downarrow 0} \frac{a\left((1-\eta) \mathbb{P}_{\theta_{0}}^{m}+\eta \mathbb{G}^{m}\right)-a\left(\mathbb{P}_{\theta_{0}}^{m}\right)}{\eta},
$$


for all $\mathbb{G}^{m}$ such that this limit exists. By contrast with a simple i.i.d. setting, in a time series framework the derivative $a^{\prime}\left(\theta_{0}, \mathbb{G}^{m}\right)$ is determined by a set of equivalent kernels. The next definition introduces a natural unique representant of such kernels.

Definition 3.1 The conditional influence function of the functional estimator $a(\cdot)$ is a kernel $I F: \mathbb{R}^{m} \times$ $\Theta \longrightarrow \mathbb{R}^{p}$ such that

i) $\quad a^{\prime}\left(\theta, \mathbb{G}^{m}\right)=\int_{\mathbb{R}^{m}} I F(y ; \theta) d \mathbb{G}^{m}(y)$, for all $\mathbb{G}^{m} \in \mathcal{M}_{\text {stat }}^{m}$,

ii) $\quad E_{\theta_{0}}\left[\operatorname{IF}\left(y_{1}^{m} ; \theta_{0}\right) \mid \mathcal{F}_{m-1}\right]=0$,

where $E_{\theta_{0}}[\cdot]$ denotes expectations with respect to the reference distribution $\mathbb{P}_{\theta_{0}}^{m}$.

Künsch (1984) introduced the natural additional condition $i$ ) which determines a unique representant of the IF. This condition simply requires that, at the reference model $\mathbb{P}_{\theta_{0}}^{m}, y_{m} \mid y_{1}, \ldots, y_{m-1}$ has no influence on the asymptotic bias of the estimator. When the dependence of the conditional IF on the corresponding score function $\psi$ has to be emphasized we use in the sequel the notation $I F_{\psi}$.

The function IF is unique (cf. also Künsch (1984), Th. 1.3) and has some desirable properties. First, under the reference model $\mathbb{P}_{\theta_{0}}^{m}$, the martingale difference property $i i$ ) implies the simple expression $V\left(\psi ; \theta_{0}\right)=E_{\theta_{0}}\left[\operatorname{IF}\left(y_{1}^{m} ; \theta_{0}\right) \operatorname{IF}\left(y_{1}^{m} ; \theta_{0}\right)^{\top}\right]$ for the asymptotic covariance matrix $V\left(\psi ; \theta_{0}\right)$ of $a(\cdot)$. Second, for conditionally unbiased $M$-estimators of the form (3) the conditional IF can be computed as in the one dimensional case by calculating the limit

$$
I F\left(x_{1}^{m} ; \theta_{0}\right):=\lim _{\eta \downarrow 0} \frac{a\left((1-\eta) \mathbb{P}_{\theta_{0}}^{m}+\eta \delta_{x_{(1)}, \ldots, x_{(m)}}\right)-a\left(\mathbb{P}_{\theta_{0}}^{m}\right)}{\eta},
$$

where $\delta_{x_{(1)}, \ldots, x_{(m)}}$ is the Dirac mass at $\left\{\left(y_{1}, \ldots, y_{m}\right)=\left(x_{(1)}, \ldots, x_{(m)}\right)\right\}$. This implies

$$
\operatorname{IF}\left(x_{1}^{m} ; a\left(\mathbb{P}_{\theta_{0}}^{m}\right)\right)=D\left(\psi ; a\left(\mathbb{P}_{\theta_{0}}^{m}\right)\right)^{-1} \psi\left(x_{1}^{m} ; a\left(\mathbb{P}_{\theta_{0}}^{m}\right)\right),
$$

where $D\left(\psi ; \theta_{0}\right):=-E_{\mathbb{P}_{\theta_{0}}^{m}}\left[(\partial / \partial \theta) \psi(y ; \theta)^{\top}\right]_{\theta=\theta_{0}}$. As the conditional IF is unique and defines a martingale difference process, (9) is the only admissible representation.

A bounded conditional IF ensures a bounded linearized asymptotic bias of any contaminated distribution $\mathbb{P}_{\eta}^{m}$ in the neighborhood $\mathcal{U}^{\eta}\left(\mathbb{P}_{\theta_{0}}^{m}\right)$

$$
\text { bias }:=a\left(\mathbb{P}_{\eta}^{m}\right)-a\left(\mathbb{P}_{\theta_{0}}^{m}\right)=\left.\eta \int_{\mathbb{R}^{m}} I F\left(y ; \theta_{0}\right) \frac{\partial}{\partial \eta} \mathbb{P}_{\eta}^{m}(d y)\right|_{\eta=0}+o\left(\left\|\mathbb{P}_{\eta}^{m}-\mathbb{P}_{\theta_{0}}^{m}\right\|\right) \text {, }
$$


because the derivative on the right hand side is uniformly bounded for any $\mathbb{P}_{\eta}^{m} \in \mathcal{U}^{\eta}\left(\mathbb{P}_{\theta_{0}}^{m}\right)$. Moreover, since the conditional IF is linearly related to the $\psi$-function of the estimating equation (4), it is bounded if and only if the $\psi$-function is bounded. As the Gaussian score function (5) is unbounded (at least) in $\varepsilon_{1}(\theta)$, PMLE's based on such a score function are not robust.

\subsection{Optimal Conditionally Unbiased Robust Estimators}

We derive the most efficient estimator with bounded self-standardized sensitivity (see Proposition 3.1 and Corollary 3.1 below) in the class of conditionally unbiased $M$-estimators for $\theta_{0}$. The self-standardized sensitivity $\gamma$ of the estimator is

$$
\gamma(\psi):=\sup _{z \in \mathbb{R}^{m}}\left\|V\left(\psi ; \theta_{0}\right)^{-1 / 2} I F_{\psi}\left(z ; \theta_{0}\right)\right\| ;
$$

cf. for instance Krasker and Welsch (1982). By definition, nonrobust estimators have $\gamma=\infty$ while bounded influence estimators have $\gamma \leq c<\infty$, for some positive constant $c \geq \sqrt{p}$; cf. Hampel et al. (1986), p. 228.

\subsubsection{Optimality Results}

Under the reference model $\mathbb{P}_{\theta_{0}}^{m}$, the classical estimator of $\theta_{0}$ defined by the score function $(5)$ is the most efficient but not robust. Therefore, we propose a robust estimator of $\theta_{0}$ that achieves for models of the form (1) an optimality result, which is the direct extension of the one in Künsch, Stefanski and Carroll (1989).

Let $\psi_{c}\left(y_{1}^{m} ; \theta\right):=A(\theta) \psi^{b i f}\left(y_{1}^{m} ; \theta\right)$, where $\psi^{b i f}\left(y_{1}^{m} ; \theta\right):=\left(s\left(y_{1}^{m} ; \theta\right)-\tau\left(y_{1}^{m-1} ; \theta\right)\right) w\left(y_{1}^{m} ; \theta\right)$. We define a robust functional $M$-estimator $\bar{a}(\cdot)$ of $\theta_{0}$ implicitly by

$$
E_{\mathbb{P}_{*}^{m}}\left[\psi_{c}\left(y_{1}^{m} ; \bar{a}\left(\mathbb{P}_{\theta_{0}}^{m}\right)\right)\right]=0
$$

where $w\left(y_{1}^{m} ; \theta\right):=\min \left(1, c\left\|A(\theta)\left(s\left(y_{1}^{m} ; \theta\right)-\tau\left(y_{1}^{m-1} ; \theta\right)\right)\right\|^{-1}\right)$. The non singular matrix $A(\theta) \in \mathbb{R}^{p} \times \mathbb{R}^{p}$ and the $\mathcal{F}_{m-1}$-measurable random vectors $\tau\left(y_{1}^{m-1} ; \theta\right) \in \mathbb{R}^{p}$ are determined by the implicit equations

$$
\begin{aligned}
& E_{\theta_{0}}\left[\psi_{c}\left(y_{1}^{m} ; \theta_{0}\right) \psi_{c}\left(y_{1}^{m} ; \theta_{0}\right)^{\top}\right]=I, \\
& E_{\theta_{0}}\left[\psi_{c}\left(y_{1}^{m} ; \theta_{0}\right) \mid \mathcal{F}_{m-1}\right]=0 .
\end{aligned}
$$


The estimating function $\psi_{c}$ (or the unscaled version $\psi^{b i f}$ ) is conditionally unbiased at the reference model and is a truncated version of the ML score (5) as, by construction, $\left\|\psi_{c}\left(y_{1}^{m} ; \theta\right)\right\| \leq c$. Moreover, as $\left(\psi_{c}\left(y_{1+t}^{m+t} ; \theta_{0}\right)\right)_{t \in \mathbb{Z}}$ is a martingale difference sequence under $\mathbb{P}_{\theta_{0}}$, the conditional IF of the functional estimator $\bar{a}(\cdot)$ is given by $(9)$,

$$
I F_{\psi_{c}}\left(y_{1}^{m} ; \bar{a}\left(\mathbb{P}_{\theta_{0}}^{m}\right)\right)=D\left(\psi_{c} ; \theta_{0}\right)^{-1} \psi_{c}\left(y_{1}^{m} ; \bar{a}\left(\mathbb{P}_{\theta_{0}}^{m}\right)\right) .
$$

The estimating function $\psi^{\text {bif }}$ satisfies the following optimality criterion.

Proposition 3.1 If for a given constant $c \geq \sqrt{p}$ equations (11) and (12) have solutions $A\left(\theta_{0}\right)$ and $\tau\left(y_{1}^{m-1} ; \theta_{0}\right)$, then $\psi^{b i f}$ minimizes $\operatorname{tr}\left(V\left(\psi ; \theta_{0}\right) V\left(\psi^{b i f} ; \theta_{0}\right)^{-1}\right)$ among all $\psi$ satisfying $(3)$ and such that

$$
\sup _{z \in \mathbb{R}^{m}}\left(I F_{\psi}\left(z ; \theta_{0}\right)^{\top} V\left(\psi^{b i f} ; \theta_{0}\right)^{-1} I F_{\psi}\left(z ; \theta_{0}\right)\right)^{1 / 2} \leq c
$$

Up to multiplication by a constant matrix, $\psi^{\text {bif }}$ is unique almost surely.

Any score function $\psi^{\text {opt }}$ such that $V\left(\psi ; \theta_{0}\right)-V\left(\psi^{\text {opt }} ; \theta_{0}\right)$ is positive semi-definite for all $\psi$ satisfying (3) is called strongly efficient. The following corollary holds.

Corollary 3.1 If there exists an unbiased, strongly efficient score function $\psi^{o p t}$ satisfying $\gamma\left(\psi^{o p t}\right) \leq c<$ $\infty$, then $\psi^{\text {opt }}$ is equivalent almost surely to $\psi^{b i f}$ whenever the latter is defined.

Proof. The proofs follow from Stefanski et al. (1986), pp. 422-423, using the property

$$
E_{\theta_{0}}\left[\tau\left(y_{1}^{m-1} ; \theta_{0}\right) \psi\left(y_{1}^{m} ; \theta_{0}\right)\right]=E_{\theta_{0}}\left[\tau\left(y_{1}^{m-1} ; \theta_{0}\right) E_{\theta_{0}}\left[\psi\left(y_{1}^{m} ; \theta_{0}\right) \mid \mathcal{F}_{m-1}\right]\right]=0
$$

for any conditionally unbiased score function $\psi$.

Under standard conditions, the optimal robust estimator $\bar{a}(\cdot)$ is consistent and asymptotically normally distributed at the reference model $\mathbb{P}_{\theta_{0}}^{m}$, with an asymptotic covariance matrix given by $V\left(\psi_{c} ; \theta_{0}\right)=$ $D\left(\psi_{c} ; \theta_{0}\right)^{-1} D\left(\psi_{c} ; \theta_{0}\right)^{-\top}$.

\subsubsection{Interpretation of $A$ and $\tau$}

The $A$ matrix ensures that the normed self-standardized IF of $\bar{a}(\cdot)$ is equal to the norm of the robust score function $\psi_{c}$, which is bounded by $c$. Indeed, under the scaling condition (11),

$$
\left\|V\left(\psi_{c} ; \theta_{0}\right)^{-1 / 2} I F_{\psi_{c}}\left(y ; \theta_{0}\right)\right\|=\left\|\psi_{c}\left(y ; \theta_{0}\right)\right\| .
$$


The $A$ matrix can be computed by a simple iterative procedure given explicitly in Section 3.2.4.

Further, to satisfy the conditional Fisher consistency condition (12), each truncated score function has to be shifted by some corresponding $\tau$-vector. This implicitly defines the random sequence of $\tau$-vectors $\left(\tau\left(y_{1+t}^{m-1+t} ; \theta_{0}\right)\right)_{t \in \mathbb{Z}}$ associated to $\left(\psi_{c}\left(y_{1+t}^{m+t} ; \theta_{0}\right)\right)_{t \in \mathbb{Z}}$. The existence of such a sequence is guaranteed by the continuity of the mapping $\tau\left(y_{1}^{m-1} ; \theta\right) \longmapsto\left(s\left(y_{1}^{m} ; \theta\right)-\tau\left(y_{1}^{m-1} ; \theta\right)\right) w\left(y_{1}^{m} ; \theta\right)$ and by the mean value theorem; cf. also Lemma 2.1 in Künsch et al. (1989). As $\tau\left(y_{1}^{m-1} ; \theta_{0}\right)$ is $\mathcal{F}_{m-1}$-measurable,

$$
\tau\left(y_{1}^{m-1} ; \theta_{0}\right)=\frac{E_{\theta_{0}}\left[s\left(y_{1}^{m} ; \theta_{0}\right) w\left(y_{1}^{m} ; \theta_{0}\right) \mid \mathcal{F}_{m-1}\right]}{E_{\theta_{0}}\left[w\left(y_{1}^{m} ; \theta_{0}\right) \mid \mathcal{F}_{m-1}\right]}
$$

In the next section we provide an accurate analytical approximation of $\tau\left(y_{1}^{m-1} ; \theta_{0}\right)$. This approximation makes use crucially of the conditionally unbiasedness of the robust score function $\psi_{c}$. For an unconditionally unbiased robust $M$-estimator the centering $\tau$-vector is implicitly defined by

$$
\tau\left(\theta_{0}\right)=\frac{E_{\theta_{0}}\left[s\left(y_{1}^{m} ; \theta_{0}\right) w\left(y_{1}^{m} ; \theta_{0}\right)\right]}{E_{\theta_{0}}\left[w\left(y_{1}^{m} ; \theta_{0}\right)\right]} .
$$

In general, the expectations in (15) cannot be expressed analytically. In these cases, the computation of $\tau$ requires computing some unconditional moments under $\mathbb{P}_{\theta_{0}}^{m}$. Unfortunately, in virtually all cases relevant for this paper such moments are unknown and the functional dependence of $\tau$ on $\theta_{0}$ and $A$ in (15) must be computed by solving some $m$-dimensional integrals by Monte Carlo simulation.

\subsubsection{Analytical Approximations for $\tau\left(y_{1}^{m-1} ; \theta_{0}\right)$}

We briefly explain the analytic approximation of the $\tau$-vectors in (14). Detailed calculations are given in Appendix A. We proceed in two steps.

Step 1. Given $\tau^{(0)}$ as initial value for $\tau\left(y_{1}^{m-1} ; \theta_{0}\right)$, we compute the real roots of the following quartic equation, with respect to the real variable $u_{m}\left(\theta_{0}\right)$,

$$
\begin{aligned}
0 & =\left\|A\left(\theta_{0}\right)\left(s\left(y_{1}^{m} ; \theta_{0}\right)-\tau^{(0)}\right)\right\|^{2}-c^{2} \\
& :=\left\|A\left(\theta_{0}\right)\left(-k_{1, m}+k_{2, m} u_{m}\left(\theta_{0}\right)+k_{1, m} u_{m}^{2}\left(\theta_{0}\right)-\tau^{(0)}\right)\right\|^{2}-c^{2}
\end{aligned}
$$

In almost all simulations and all empirical estimations in Sections 5 and 6, equation (16) had only two real roots. Therefore, we only consider this case for brevity. The case of four real roots is discussed in Appendix A. 
Step 2. We 'split' the integrals in (14) according to the roots determined by (16). Denoting such roots by $\underline{u}_{m}$ and $\bar{u}_{m}$, with $\underline{u}_{m} \leq \bar{u}_{m}$, the denominator in (14) is

$$
\begin{aligned}
& E_{\theta_{0}}\left[w\left(y_{1}^{m} ; \theta_{0}\right) \mid \mathcal{F}_{m-1}\right]= \\
& \quad \int_{-\infty}^{\underline{u}_{m}} \frac{c}{\left\|A\left(\theta_{0}\right)\left(s\left(v ; \theta_{0}\right)-\tau^{(0)}\right)\right\|} d \Phi(u)+\left[\Phi\left(\bar{u}_{m}\right)-\Phi\left(\underline{u}_{m}\right)\right]+\int_{\bar{u}_{m}}^{+\infty} \frac{c}{\left\|A\left(\theta_{0}\right)\left(s\left(v ; \theta_{0}\right)-\tau^{(0)}\right)\right\|} d \Phi(u),
\end{aligned}
$$

where $v:=\left(y_{1}^{m-1}, \mu_{m}\left(\theta_{0}\right)+\sigma_{m}\left(\theta_{0}\right) u\right)$ and $\phi(\cdot)$ and $\Phi(\cdot)$ denote the standard Gaussian density and cumulative distribution function. In our applications, typical values of $\bar{u}_{m}$ range from 2.7 to 3.5 (the opposite for $\left.\underline{u}_{m}\right)$. Hence, the 'main contribution' to the expectation on the left hand side of (17) comes from the term in the square brackets. Since $\underline{u}_{m}$ and $\bar{u}_{m}$ are 'quite far' in the tails of a standard Gaussian distribution, the remaining integrals can be well approximated using Laplace's method; cf. for instance Jensen (1995), Th. 3.1.1. The integral in the numerator of (14) is split in the same way as in (17) and the relevant integrals are again approximated using Laplace's method. The resulting formula for the computation of $\tau$ is given in the next proposition.

Proposition 3.2 Given model (1) and the conditionally Gaussian reference model $\mathbb{P}_{\theta_{0}}^{m}$, if the quartic equation (16) has only two real roots $\underline{u}_{m} \leq \bar{u}_{m}$, then

$\tau\left(y_{1}^{m-1} ; \theta_{0}\right)=\frac{-L_{n}\left(\underline{u}_{m}\right)-k_{1, m}\left[\Phi\left(\bar{u}_{m}\right)-\Phi\left(\underline{u}_{m}\right)\right]+k_{2, m} M_{1, m}+k_{1, m} M_{2, m}+L_{n}\left(\bar{u}_{m}\right)}{-L_{d}\left(\underline{u}_{m}\right)+\left[\Phi\left(\bar{u}_{m}\right)-\Phi\left(\underline{u}_{m}\right)\right]+L_{d}\left(\bar{u}_{m}\right)}+O\left(\underline{u}_{m}^{-3}\right)+O\left(\bar{u}_{m}^{-3}\right)$

where $M_{1, m}:=\phi\left(\underline{u}_{m}\right)-\phi\left(\bar{u}_{m}\right), M_{2, m}:=\underline{u}_{m} \phi\left(\underline{u}_{m}\right)-\bar{u}_{m} \phi\left(\bar{u}_{m}\right)+\Phi\left(\bar{u}_{m}\right)-\Phi\left(\underline{u}_{m}\right) . L_{n}(\cdot)$ and $L_{d}(\cdot)$ are defined in Appendix A and correspond to some Laplace approximations for the integrals in the numerator and in the denominator of (14).

Intuitively, the real roots $\underline{u}_{m}$ and $\bar{u}_{m}$ in equation (16) determine the range where the standardized innovation $u_{m}\left(\theta_{0}\right)$ is 'not influential' (in terms of the self-standardized sensitivity of $\bar{a}(\cdot)$ ) for the arising asymptotic bias. Indeed,

$$
\begin{aligned}
\left\|A\left(\theta_{0}\right)\left(s\left(y_{1}^{m} ; \theta_{0}\right)-\tau^{(0)}\right)\right\| \leq c, \Longleftrightarrow u_{m}\left(\theta_{0}\right) \in\left[\underline{u}_{m}, \bar{u}_{m}\right], \\
>c, \Longleftrightarrow u_{m}\left(\theta_{0}\right) \in\left(-\infty, \underline{u}_{m}\right) \cup\left(\bar{u}_{m},+\infty\right),
\end{aligned}
$$

and the normed self-standardized IF of $\bar{a}(\cdot)$ is equal to the norm of the $\psi_{c}$-function. 


\subsubsection{Algorithm}

To compute the robust estimator defined by (10)-(12) an iterative algorithm has to be adopted because the weights $w\left(y_{1}^{m} ; \bar{a}\left(\mathbb{P}_{\theta_{0}}^{m}\right)\right)$, the matrix $A\left(\bar{a}\left(\mathbb{P}_{\theta_{0}}^{m}\right)\right)$ and the random vectors $\tau\left(y_{1}^{m-1} ; \bar{a}\left(\mathbb{P}_{\theta_{0}}^{m}\right)\right)$ depend on the value of the estimator itself in a nonlinear way. Given a constant $c \geq \sqrt{p}$ (cf. Hampel et al. (1986), p. 228), the robust estimator is computed by the following four steps algorithm.

1. Fix a starting value $\theta^{(0)}$ for $\theta_{0}$, and initial values $\tau_{t}^{(0)}:=\tau\left(\tilde{y}_{t-m+1}^{t-1} ; \theta^{(0)}\right)=0$, for all $t=1, \ldots, n$ and $A^{(0)}$ such that

$$
A^{(0)^{\top}} A^{(0)}=\left[n^{-1} \sum_{t=1}^{n} s\left(\tilde{y}_{t-m+1}^{t} ; \theta^{(0)}\right) s\left(\tilde{y}_{t-m+1}^{t} ; \theta^{(0)}\right)^{\top}\right]^{-1} .
$$

2. Compute, for all $t=1, \ldots, n$, the real roots of equations (16), and the associated new values $\tau_{t}^{(1)}:=\tau\left(\tilde{y}_{t-m+1}^{t-1} ; \theta^{(0)}\right)$ for $\tau_{t}$ and the new matrix $A^{(1)}$ for $A$, defined by

$$
\begin{gathered}
\tau_{t}^{(1)}:=\frac{-L_{n}\left(\underline{u}_{t}\right)-k_{1, t}\left[\Phi\left(\bar{u}_{t}\right)-\Phi\left(\underline{u}_{t}\right)\right]+k_{2, t} M_{1, t}+k_{1, t} M_{2, t}+L_{n}\left(\bar{u}_{t}\right)}{-L_{d}\left(\underline{u}_{t}\right)+\left[\Phi\left(\bar{u}_{t}\right)-\Phi\left(\underline{u}_{t}\right)\right]+L_{d}\left(\bar{u}_{t}\right)}, \\
\left(A^{(1)^{\top}} A^{(1)}\right)^{-1}:=n^{-1} \sum_{t=1}^{n}\left(s\left(\tilde{y}_{t-m+1}^{t} ; \theta^{(0)}\right)-\tau_{t}^{(0)}\right)\left(s\left(\tilde{y}_{t-m+1}^{t} ; \theta^{(0)}\right)-\tau_{t}^{(0)}\right)^{\top} \times \\
\min ^{2}\left(1, c\left\|A^{(0)}\left(s\left(\tilde{y}_{t-m+1}^{t} ; \theta^{(0)}\right)-\tau^{(0)}\right)\right\|^{-1}\right) .
\end{gathered}
$$

3. Compute the robust estimator $\theta^{(1)}$ implied by (10) for given $A^{(1)}$ and $\tau_{t}^{(1)}$ as the solution of the implicit equation

$$
\sum_{t=1}^{n}\left(s\left(\tilde{y}_{t-m+1}^{t} ; \theta^{(1)}\right)-\tau_{t}^{(1)}\right) \min \left(1, c\left\|A^{(1)}\left(s\left(\tilde{y}_{t-m+1}^{t} ; \theta^{(0)}\right)-\tau_{t}^{(1)}\right)\right\|^{-1}\right)=0 .
$$

4. Replace $A^{(0)}$ by $A^{(1)}$ and $\tau_{t}^{(0)}$ by $\tau_{t}^{(1)}$ for all $t=1, \ldots, n$ and iterate steps 2 and 3 above until convergence of the sequence $\left(\theta^{(i)}\right)_{i \in \mathbb{N}}$ of estimators associated with (10) and with the sequence $\left(A^{(i)}, \bar{\tau}^{(i)}\right)_{i \in \mathbb{N}}$, where $\bar{\tau}^{(i)}:=\left(\tau_{1}^{(i)}, \ldots, \tau_{n}^{(i)}\right)$.

Starting values for $\theta^{(0)}$ could be the PML estimate of $\theta_{0}$ or the result of a grid search algorithm. We wrote a Matlab code to implement the algorithm and we used the Matlab function 'roots' to compute the real roots of equation (16). Analytical expressions for the $\tau$-vectors avoid "internal" simulations to compute the robust estimator. This largely reduces the computation time. For comparison, we implemented a 
second algorithm in which the $\tau$ integrals were computed numerically using the Matlab function 'quadl'. This algorithm is unfeasible as the computation time of $\bar{a}$ is almost two hours already for a simple AR(1)$\mathrm{ARCH}(1)$ model. For further comparison, we also implemented a robust GMM estimator as in Ronchetti and Trojani (2001) with moment conditions $A(\theta)\left(s\left(y_{1}^{m} ; \theta\right)-\tau(\theta)\right) w\left(y_{1}^{m} ; \theta\right)$, where $\tau(\theta)$ is given by (15). In our simulations of Section 5, the computation time of the estimator (10)-(12) was about $20 \%$ the one of such a robust GMM estimator.

\section{Robust Testing Procedures}

Robust versions of the classical Wald, score and likelihood ratio tests based on the robust estimator in (10)-(12) can be derived following the general approach proposed by Heritier and Ronchetti (1994) and Ronchetti and Trojani (2001). Such robust tests satisfy the optimality criterion of maximizing the asymptotic power subject to a bound on the asymptotic bias of the level and the power test. Such biases can be controlled by imposing a bound on $\gamma$; cf. Ronchetti and Trojani (2001), p. 54. For brevity, we focus on the robust version of the Wald test. Robust score and likelihood ratio tests can be handled in a similar way.

Consider a parametric null hypothesis of the form

$$
g\left(\bar{a}\left(\mathbb{P}_{\theta_{0}}^{m}\right)\right)=0
$$

for a smooth function $g: \Theta \longrightarrow \mathbb{R}^{r}$ such that $(\partial / \partial \bar{a}) g\left(\bar{a}\left(\mathbb{P}_{\theta}^{m}\right)\right)^{\top}$ is of full column rank $r$ for all $\theta \in \Theta$.

We consider for any $n \in \mathbb{N}$ test statistics $n Q$ that are quadratic forms of a Wald functional $U$,

$$
n Q\left(\mathbb{P}_{n}^{m}\right):=n U\left(\mathbb{P}_{n}^{m}\right)^{\top} U\left(\mathbb{P}_{n}^{m}\right) ; \quad U\left(\mathbb{P}_{n}^{m}\right):=\left[\frac{\partial g(\theta)}{\partial \theta^{\top}} V\left(\psi_{c} ; \theta\right) \frac{\partial g(\theta)^{\top}}{\partial \theta}\right]_{\theta=\bar{a}\left(\mathbb{P}_{n}^{m}\right)}^{-1 / 2} g\left(\bar{a}\left(\mathbb{P}_{n}^{m}\right)\right),
$$

where $\mathbb{P}_{n}^{m}$ is the empirical $m$-dimensional distribution of the observations $\tilde{y}_{1}^{n}$. Under the reference model $\mathbb{P}_{\theta_{0}}^{m}$ and the null hypothesis (18), $n Q\left(\mathbb{P}_{n}^{m}\right)$ converges in distribution to a $\chi^{2}$ distribution with $r$ degrees of freedom. To apply the methodology in Heritier and Ronchetti (1994), we make the following assumption.

Assumption 4.1 Let a bounded-influence estimator $\bar{a}$ of $a\left(\mathbb{P}_{\theta_{0}}^{m}\right)$ be given. Then,

$$
\sqrt{n}\left(\bar{a}\left(\mathbb{P}_{n}^{m}\right)-\bar{a}\left(\mathbb{P}_{\eta(\epsilon, n)}^{m}\right)\right) \rightarrow \mathcal{N}\left(0, V\left(\psi ; \theta_{0}\right)\right), n \rightarrow \infty
$$


in distribution, uniformly over the sequence $\left(\mathcal{U}^{\eta(\epsilon, n)}\left(\mathbb{P}_{\theta_{0}}^{m}\right)\right)_{n \in \mathbb{N}}$ of $\eta(\epsilon, n)$-neighborhoods defined by (7) for $\eta:=\eta(\epsilon, n)=\epsilon / \sqrt{n}$ and $\mathbb{G} \in \operatorname{dom}(\bar{a})$.

Under Assumption 4.1 the following proposition holds.

Proposition 4.1 Let $\bar{a}$ be the robust estimator defined by (10)-(12) and denote by $\alpha$ the asymptotic level functional of the Wald statistic (19). Further let $\left(\mathbb{P}_{\eta(\epsilon, n)}^{m}\right)_{n \in \mathbb{N}}$ be a sequence of $\eta(\epsilon, n)$-contaminations of the null distribution $\mathbb{P}_{\theta_{0}}^{m}$, i.e. $\mathbb{P}_{\eta(\epsilon, n)}^{m} \in \mathcal{U}^{\eta(\epsilon, n)}\left(\mathbb{P}_{\theta_{0}}^{m}\right)$. Then, the bias of the asymptotic level $\lim _{n \rightarrow \infty} \alpha\left(\mathbb{P}_{\eta(\epsilon, n)}^{m}\right)$ is uniformly bounded by

$$
\lim _{n \rightarrow \infty}\left|\alpha\left(\mathbb{P}_{\eta(\epsilon, n)}^{m}\right)-\alpha_{0}\right| \leq \epsilon^{2} \mu \sup _{z \in \mathbb{R}^{m}}\left\|\psi_{c}(z ; \bar{a})\right\|^{2}+o\left(\epsilon^{2}\right)
$$

where $\mu:=-\left.(\partial / \partial \beta) H_{r}\left(q_{1-\alpha_{0}} ; \beta\right)\right|_{\beta=0}, H_{r}(\cdot ; \beta)$ is the cumulative distribution function of a noncentral $\chi^{2}(r ; \beta)$ distribution with $r$ degrees of freedom and noncentrality parameter $\beta \geq 0, q_{1-\alpha_{0}}$ is the $1-\alpha_{0}$ quantile of a $\chi^{2}(r ; 0)$ distribution and $\alpha_{0}=\alpha\left(\mathbb{P}_{\theta_{0}}^{m}\right)$ is the nominal asymptotic level of the test at the reference model.

Proof. The proof follows from Heritier and Ronchetti (1994), p. 903 and Ronchetti and Trojani (2001), p. 64 .

Interpretion of $\varepsilon$. Choice of $c$. As a consequence of Proposition 4.1, the maximal asymptotic bias in the level of the test based on the robust $M$-estimator $\bar{a}$ in (10)-(12) is bounded by

$$
\lim _{n \rightarrow \infty}\left|\alpha\left(\mathbb{P}_{\eta(\epsilon, n)}^{m}\right)-\alpha_{0}\right| \leq \mu(\epsilon c)^{2}+o\left(\epsilon^{2}\right)
$$

The "power" counterpart of Proposition 4.1 can also be obtained. Hence, also the maximal asymptotic bias in the power of a robust Wald test can be controlled by our robust estimator $\bar{a}$; cf. Ronchetti and Trojani (2001), Th. 2 .

\section{Monte Carlo Simulations}

We compare by Monte Carlo simulations the performance of the classical PMLE (cf. Gourieroux, Monfort and Trognon (1984)) defined by the score function (5) with the one of our robust estimator, both at the 
reference model and in the presence of local model contaminations. We estimate an $\mathrm{AR}(1)-\mathrm{ARCH}(1)$ model and simulate the following contaminated models "near" the reference model $\mathbb{P}_{\theta_{0}}$.

1. Standard Gaussian innovations. In this experiment, the innovation $u_{t}\left(\theta_{0}\right)$ has a standard Gaussian distribution. Hence, the PMLE is the MLE and we compare the efficiency of our robust estimator and the MLE at the reference model $\mathbb{P}_{\theta_{0}}$.

2. Replacement model (cf. for instance Martin and Yohai (1986)). Under such a model the observed process $\mathcal{X}:=\left(x_{t}\right)_{t \in \mathbb{Z}}$ is generated according to the data generating process,

$$
x_{t}=\left(1-\vartheta_{t}^{\eta}\right) y_{t}+\vartheta_{t}^{\eta} \xi_{t},
$$

where the clean process $\mathcal{Y}:=\left(y_{t}\right)_{t \in \mathbb{Z}}$ is generated by the reference model $\mathbb{P}_{\theta_{0}}$ and $\left(\vartheta_{t}^{\eta}\right)_{t \in \mathbb{Z}}$ is an i.i.d. 0 -1 random sequence, independent of $\mathcal{Y}$, such that $\mathbb{P}\left(\vartheta_{t}^{\eta}=1\right)=\eta$. Hence, at a time $t \in \mathbb{Z}$, the clean observation $y_{t}$ is replaced by $\xi_{t}$ with probability $\eta$. In our simulations we set $\eta=0.5 \%$ and $\xi_{t}=1.5$ for all $t$. Such a low probability of contaminations is motivated by some difficulties of the standard PMLE to converge when higher probabilities of contaminations occur (for e.g. $\eta=1 \%$ ). In this experiment model (1) is dynamically "slightly" misspecified as the dynamic equations (1) are not satisfied. This experiment allows to compare the performances of the PMLE and the robust estimator when very few observations deviate from the assumed model.

3. Innovative outlier model (cf. for instance Bustos and Yohai (1986)). Under such a contamination the innovations are given by $u_{t}\left(\theta_{0}\right)=\check{u}_{t}\left(\theta_{0}\right)\left[(1-\epsilon)+\epsilon \varrho^{2}\right]^{-1 / 2}$, where $\check{u}_{t}\left(\theta_{0}\right)$ is distributed as the following mixture distribution

$$
\check{u}_{t}\left(\theta_{0}\right) \sim(1-\epsilon) \mathcal{N}(0,1)+\epsilon \mathcal{N}\left(0, \varrho^{2}\right) .
$$

We set $\epsilon=1 \%$ and $\varrho=3$. (22) describes situations where a given shock (or outlier) affects also future realizations of the process $\mathcal{Y}$. Furthermore, as $u_{t}\left(\theta_{0}\right) \sim$ i.i.d. $(0,1)$, the dynamic equations $(1)$ are satisfied and the model is dynamically correctly specified. Hence, this is a typical situation in which the PMLE is applied and there are no theoretical efficiency reasons to prefer one estimator to the other. 
We simulate an $\operatorname{AR}(1)-\operatorname{ARCH}(1)$ model implied by (2) for the parameter choice: $\rho_{0}=0.01, \rho_{1}=0.8$, $\alpha_{0}=0.02, \alpha_{1}=0.8$ and $\rho_{2}=\alpha_{2}=\alpha_{3}=0$ under the above distributions for $y_{t}$ and for a sample size $n=1,000$. The tuning constant for the robust estimator $\bar{a}$ was set at $c=9$. Such a rather large value of the tuning constant $c$ implies that very few observations were downweighted. For instance, in our simulations under the reference model $\mathbb{P}_{\theta_{0}}$ only 3 or 4 (out of 1,000 ) observations were typically slightly downweighted with weights around 0.8-0.9. Each model is simulated 5,000 times. For each simulation we compute the PML and the robust estimates of $\theta_{0}$ with the corresponding asymptotic covariance matrices. Then, for each parameter we compute the corresponding confidence interval at the $95 \%$ confidence level.

In a simulation study not reported here we also compared the performances of the RGMM estimates introduced in Section 3.2.4 and our robust estimator under the reference model $\mathbb{P}_{\theta_{0}}$ and the replacement model (21) for $\eta=5 \%, \xi_{t} \sim \mathcal{N}(0,1)$ and $c=4$ for both estimators. The two performances were quite close, up to the large differences in the computation time.

\subsection{Point Estimation}

Estimation results are presented in Table 1. For each estimated parameter, the first (second) column contains summary statistics for the PML estimates (the robust estimates). In Figures 1-3 we plot the estimated densities of the classical and the robust estimators. The first panel in Table 1 shows that the efficiency loss of the robust estimator at the reference model $\mathbb{P}_{\theta_{0}}$ is almost negligible. Specifically, the mean squared errors of all parameter estimates for the two estimation procedures are very close. This is confirmed by Figure 1. The second panel in Table 1 and Figure 2 show instead large biases and mean squared errors of PML estimates under the replacement model (21). By contrast, robust estimates maintain low mean squared errors. It is somehow surprising that such a large bias in the PML estimates is induced by contaminating (on average) only $0.5 \%$ of the sample. Finally, the third panel in Table 1 and Figure 3 show that, in terms of mean squared error, both estimators estimate correctly the conditional mean parameters $\rho_{0}$ and $\rho_{1}$. However, the robust estimator always outperforms the PMLE in the estimation of the conditional variance parameters $\alpha_{0}$ and $\alpha_{1}$. 


\subsection{Interval Estimation}

Figures 4-6 show the boxplots of the estimated confidence interval lengths for the PML and the robust estimates. Actual confidence interval coverages are close to the nominal level $95 \%$ in both cases (an exception is the confidence interval of the parameter $\alpha_{0}$ which is $78 \%$ for the PMLE and $92 \%$ for our robust estimator under the replacement model (21).) Moreover, Figure 4 shows that, under the reference model $\mathbb{P}_{\theta_{0}}$, the confidence interval lengths for both estimation techniques are almost identical. However, Figure 5 shows that, under the replacement model (21), the PML confidence intervals are much larger than the robust ones, denoting a large inaccuracy of the inference results. Moreover, PML confidence intervals are not centered around $\theta_{0}$ as the parameter estimates are biased; cf. again Figure 2. Robust confidence intervals are much more concentrated around $\theta_{0}$. Finally, Figure 6 shows that confidence intervals under the innovative outlier model (22) are tighter for the robust than for the PML estimates, especially for the conditional variance parameters $\alpha_{0}$ and $\alpha_{1}$.

\subsection{Hypothesis Testing}

To compare the performance of the classical PMLE and our robust estimator from the perspective of hypothesis testing we also simulated 1,000 sample paths of an $\mathrm{AR}(1)-\mathrm{ARCH}(1)$ model for the parameter choices $\rho_{0}=0, \rho_{1}=0,0.05,0.10$ and $\alpha_{0}=0.02, \alpha_{1}=0.8$ under scaled Student $t_{3}$ and scaled Student $t_{5}$ innovations. We do not necessarily believe that in applications the innovations will generally follow these distributions. We rather take the student $t_{3}$ and $t_{5}$ distributions as further examples of distributions which are very close to the normal one. Under scaled Student $t$ innovations model (1) is dynamically correctly specified and hence the PMLE should perform well. In our experiments we tested the joint null hypothesis $\rho_{0}=\rho_{1}=0$ by means of a classical and a robust Wald statistic. The empirical rejection frequencies of a Wald test based on the classical PMLE and a Wald test based on our robust estimator are calculated for a fixed nominal level $5 \%$ of the test. The results are presented in Table 2. The estimated standard error of the empirical rejection frequency $\hat{p}$ is $0.7 \%, 1.4 \%, 1.5 \%$ for $\hat{p}=5 \%, 30 \%$, $60 \%$, respectively. Table 2 shows that the robust Wald test performs very well across all models, while the classical test is oversized in finite sample and shows a lower power than the robust ones. 
The low power of classical tests under even slight departures from conditional normality suggests that robust tests could be useful in application to unmask some possible 'dynamics' in the data hidden by the presence of influential observations.

\section{Empirical Application}

We apply classical and robust Wald tests for $\mathrm{ARCH}$ to weekly exchange rate returns of the Spanish peseta against the US dollar over the period November $2^{\text {nd }}, 1993$ until October $28^{\text {th }}, 2003$. The data were downloaded from Datastream and consist of 522 observations. A similar data set from the Federal Reserve Bank of Chicago has been analyzed by van Dijk et al. (1999) over the sample period January $8^{\text {th }}$, 1986 until December $27^{\text {th }}, 1995$. Our sample period contains a more 'regular' time series where no large clear outlier seem to occur in the data. Indeed, the first ten sample autocorrelations of squared and absolute returns are not significantly different from zero. Moreover, the Jarque-Bera test has a $p$-value of 0.54 not rejecting normality. Classical PML estimates for the parameters $\rho_{0}, \rho_{1}, \alpha_{0}$ and $\alpha_{1}$ of an $\mathrm{AR}(1)-\mathrm{ARCH}(1)$ model (Wald test $p$-values for the hypothesis that the corresponding parameter is zero) are $0.02(0.76), 0.006(0.90), 1.67(0), 0.04(0.55)$. The robust estimates under a tuning constant $c=4$ are $0.04(0.50), 0.001(0.97), 1.54(0), 0.44\left(3.9 \cdot 10^{-5}\right)$. Therefore, as is typical for many financial return series, the conditional mean parameters are not significantly different from zero. Moreover, the PML estimate of the $\mathrm{ARCH}$ parameter $\alpha_{1}$ is also not significant. Hence, the classical Wald test does not reject the homoscedasticity hypothesis. By contrast, the robust estimate of this ARCH parameter is highly significant, showing that $\mathrm{ARCH}$ effects in the data are possibly obscured by some outlying observations detected by the robust Huber weight presented in the bottom panel of Figure 8. These results are consistent with the low power of PML tests under nonnormal conditional returns in Section 5.3.

\section{Conclusions}

We derived optimal bounded-influence estimators for the parameters of conditional location and scale models under a conditionally Gaussian reference model. Based on these results, we obtained optimal 
bounded-influence versions of the classical likelihood-based tests for parametric hypotheses. We proposed an efficient algorithm for the computation of our robust estimators, which strongly reduces the necessary computation time by avoiding the simulation of multidimensional integrals. Monte Carlo simulations show that our robust estimators maintain a high efficiency under ideal model conditions and have good robustness properties under local departures from conditional normality, both in estimation and inference. On the contrary, classical PML estimators are strongly biased and highly inefficient even under small departures from conditional Gaussianity. An application to exchange rate data seems to confirm these patterns. 


\section{A Computation of $\tau\left(y_{1}^{m-1} ; \theta_{0}\right)$}

This appendix describes the computation of the correction factor $\tau\left(y_{1}^{m-1} ; \theta_{0}\right)$ in equation (12). According to (16), the Gaussian score function can be written as

$$
s\left(y_{1}^{m} ; \theta_{0}\right)=-k_{1, m}+k_{2, m} u_{m}\left(\theta_{0}\right)+k_{1, m} u_{m}^{2}\left(\theta_{0}\right)
$$

where $u_{m}\left(\theta_{0}\right) \sim \mathcal{N}(0,1)$ under the reference model $\mathbb{P}_{\theta_{0}}^{m}$. For brevity we write $A$ instead of $A\left(\theta_{0}\right)$. Formally, the problem is to compute $\tau\left(y_{1}^{m-1} ; \theta_{0}\right)$ such that

$$
0=A \int_{-\infty}^{+\infty}\left(-k_{1, m}+k_{2, m} u+k_{1, m} u^{2}-\tau\left(y_{1}^{m-1} ; \theta_{0}\right)\right) w\left(y_{1}^{m-1}, \mu_{m}\left(\theta_{0}\right)+\sigma_{m}\left(\theta_{0}\right) u ; \theta_{0}\right) d \Phi(u) .
$$

As $\tau\left(y_{1}^{m-1} ; \theta_{0}\right), k_{1, m}$ and $k_{2, m}$ are $\mathcal{F}_{m-1}$-measurable, we have

$$
\tau\left(y_{1}^{m-1} ; \theta_{0}\right)=\frac{\tau_{\text {num }}\left(y_{1}^{m-1} ; \theta_{0}\right)}{\tau_{\text {den }}\left(y_{1}^{m-1} ; \theta_{0}\right)}
$$

where

$$
\begin{aligned}
& \tau_{\text {num }}\left(y_{1}^{m-1} ; \theta_{0}\right):=\int_{-\infty}^{+\infty}\left(-k_{1, m}+k_{2, m} u+k_{1, m} u^{2}\right) w\left(y_{1}^{m-1}, \mu_{m}\left(\theta_{0}\right)+\sigma_{m}\left(\theta_{0}\right) u ; \theta_{0}\right) d \Phi(u), \\
& \tau_{d e n}\left(y_{1}^{m-1} ; \theta_{0}\right):=\int_{-\infty}^{+\infty} w\left(y_{1}^{m-1}, \mu_{m}\left(\theta_{0}\right)+\sigma_{m}\left(\theta_{0}\right) u ; \theta_{0}\right) d \Phi(u) .
\end{aligned}
$$

Clearly, the difficulties in the computation of these integrals derive from the presence of the weighting function $w\left(y_{1}^{m} ; \theta_{0}\right)$ defined by $(10)$. However, as the weighting function implies that $\left\|\psi_{c}\left(y_{1}^{m} ; \theta_{0}\right)\right\|^{2} \leq$ $c^{2}$, we can equivalently express such an inequality in terms of 'admissible' values of the standardized innovation $u_{m}\left(\theta_{0}\right)$. Specifically, we compute $\tau\left(y_{1}^{m-1} ; \theta_{0}\right)$ by means of the following two steps procedure.

\section{Step 1}

In the first step we compute the real roots in the real variable $u_{m}\left(\theta_{0}\right)$ of the quartic equation (16), i.e.

$$
\begin{aligned}
0 & =\left\|A\left(s\left(y_{1}^{m} ; \theta_{0}\right)-\tau^{(0)}\right)\right\|^{2}-c^{2} \\
& =\left\|A\left(-k_{1, m}+k_{2, m} u_{m}\left(\theta_{0}\right)+k_{1, m} u_{m}^{2}\left(\theta_{0}\right)-\tau^{(0)}\right)\right\|^{2}-c^{2} \\
& =a_{4} u_{m}^{4}\left(\theta_{0}\right)+a_{3} u_{m}^{3}\left(\theta_{0}\right)+a_{2} u_{m}^{2}\left(\theta_{0}\right)+a_{1} u_{m}\left(\theta_{0}\right)+a_{0}-c^{2},
\end{aligned}
$$

where

$$
a_{4}:=k_{1, m}^{\top} A^{\top} A k_{1, m}, \quad a_{3}:=2 k_{1, m}^{\top} A^{\top} A k_{2, m},
$$




$$
\begin{aligned}
& a_{2}:=k_{2, m}^{\top} A^{\top} A k_{2, m}-2 k_{1, m}^{\top} A^{\top} A k_{1, m}-2 k_{1, m}^{\top} A^{\top} A \tau^{(0)}, \\
& a_{1}:=-a_{3}-2 k_{2, m}^{\top} A^{\top} A \tau^{(0)}, \\
& a_{0}:=a_{4}+2 k_{1, m}^{\top} A^{\top} A \tau^{(0)}+\tau^{(0) \top} A^{\top} A \tau^{(0)} .
\end{aligned}
$$

Existence of a solution is guaranteed by Lemma 2.1 in Künsch et al. (1989) when choosing $c \geq \sqrt{p}$. In general, we have either two or four real roots. As $a_{4}>0$, in the first case

$$
\begin{aligned}
\left\|A\left(s\left(y_{1}^{m} ; \theta_{0}\right)-\tau^{(0)}\right)\right\| & \leq c, u_{m}\left(\theta_{0}\right) \in\left[\underline{u}_{m}, \bar{u}_{m}\right] \\
& >c, \quad u_{m}\left(\theta_{0}\right) \in\left(-\infty, \underline{u}_{m}\right) \cup\left(\bar{u}_{m},+\infty\right),
\end{aligned}
$$

denoting by $\underline{u}_{m} \leq \bar{u}_{m}$ the real roots. In the second case, with four real roots $\underline{\underline{u}}_{m} \leq \underline{u}_{m} \leq \bar{u}_{m} \leq \overline{\bar{u}}_{m}$,

$$
\begin{aligned}
\left\|A\left(s\left(y_{1}^{m} ; \theta_{0}\right)-\tau^{(0)}\right)\right\| & \leq c, u_{m}\left(\theta_{0}\right) \in\left[\underline{\underline{u}}_{m}, \underline{u}_{m}\right] \cup\left[\bar{u}_{m}, \overline{\bar{u}}_{m}\right] \\
& >c, u_{m}\left(\theta_{0}\right) \in\left(-\infty, \underline{\underline{u}}_{m}\right) \cup\left(\underline{u}_{m}, \bar{u}_{m}\right) \cup\left(\overline{\bar{u}}_{m},+\infty\right) .
\end{aligned}
$$

In almost all simulations and all empirical estimations in the paper we found only two real roots.

\section{Step 2}

In the second step we 'split' the integral in equation (23) and (24) according to the roots determined in

Step 1. Assume first that there are two real roots, then

$$
\begin{aligned}
\tau_{\text {num }}( & \left.y_{1}^{m-1} ; \theta_{0}\right) \\
= & \int_{-\infty}^{\underline{u}_{m}} \overbrace{\left(-k_{1, m}+k_{2, m} u+k_{1, m} u^{2}\right) \frac{c}{\left\|A\left(s\left(v ; \theta_{0}\right)-\tau^{(0)}\right)\right\|}}^{q_{n}(u):=} d \Phi(u) \\
& +\int_{\underline{u}_{m}}^{\bar{u}_{m}}\left(-k_{1, m}+k_{2, m} u+k_{1, m} u^{2}\right) d \Phi(u) \\
& +\int_{\bar{u}_{m}}^{+\infty}\left(-k_{1, m}+k_{2, m} u+k_{1, m} u^{2}\right) \frac{c}{\left\|A\left(s\left(v ; \theta_{0}\right)-\tau^{(0)}\right)\right\|} d \Phi(u) \\
:= & \int_{-\infty}^{\underline{u}_{m}} q_{n}(u) \frac{1}{\sqrt{2 \pi}} \exp \left(-.5 u^{2}\right) d u-k_{1, m}\left[\Phi\left(\bar{u}_{m}\right)-\Phi\left(\underline{u}_{m}\right)\right]+k_{2, m} M_{1, m}+k_{1, m} M_{2, m} \\
& +\int_{\bar{u}_{m}}^{+\infty} q_{n}(u) \frac{1}{\sqrt{2 \pi}} \exp \left(-.5 u^{2}\right) d u .
\end{aligned}
$$

Notice that $q_{n}: \mathbb{R} \longrightarrow \mathbb{R}^{p}$ with the same functional form in each component. $M_{1, m}, M_{2, m}$ are defined in Proposition 3.2 and $v:=\left(y_{1}^{m-1}, \mu_{m}\left(\theta_{0}\right)+\sigma_{m}\left(\theta_{0}\right) u\right)$.

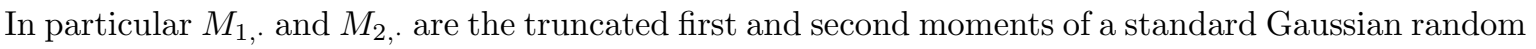


variable and integration by parts yields

$$
M_{1, .}=\int_{a}^{b} u d \Phi(u)=\phi(a)-\phi(b), \quad M_{2, .}=\int_{a}^{b} u^{2} d \Phi(u)=a \phi(a)-b \phi(b)+\Phi(b)-\Phi(a) .
$$

The remaining univariate integrals are approximated 'componentwise' using Laplace's method. Under standard regularity conditions (cf. for instance Jensen (1995), p. 58) on the real function $q(\cdot)$, for $\alpha \rightarrow \infty$

$$
\begin{aligned}
\int_{0}^{\infty} \alpha \exp (-\alpha u) q(u) d u & =q(0)+\frac{q^{\prime}(0)}{\alpha}+\frac{q^{\prime \prime}(0)}{\alpha^{2}}+O\left(\frac{1}{\alpha^{3}}\right) \\
& =: \quad \mathcal{L}(q, \alpha)+O\left(\frac{1}{\alpha^{3}}\right) .
\end{aligned}
$$

$\mathcal{L}(q, \alpha)$ is the Laplace approximation of the integral up to the third order. We use third order Laplace approximations as the contribution of higher order terms is negligible. Therefore,

$$
\begin{aligned}
\int_{\bar{u}_{m}}^{+\infty} & q_{n}(u) \frac{1}{\sqrt{2 \pi}} \exp \left(-.5 u^{2}\right) d u \\
= & \frac{1}{\sqrt{2 \pi}} \exp \left(-.5 \bar{u}_{m}^{2}\right) \frac{1}{\bar{u}_{m}} \int_{0}^{+\infty} \bar{u}_{m} \exp \left(-\bar{u}_{m} z\right) q_{n}\left(\bar{u}_{m}+z\right) \exp \left(-.5 z^{2}\right) d z \\
= & \frac{1}{\sqrt{2 \pi}} \exp \left(-.5 \bar{u}_{m}^{2}\right) \frac{1}{\bar{u}_{m}}\left(\mathcal{L}\left(\bar{q}_{n}, \bar{u}_{m}\right)+O\left(\frac{1}{\bar{u}_{m}^{3}}\right)\right) \\
=: & L_{n}\left(\bar{u}_{m}\right)+O\left(\frac{1}{\bar{u}_{m}^{3}}\right),
\end{aligned}
$$

where $\bar{q}_{n}(z):=q_{n}\left(\bar{u}_{m}+z\right) \exp \left(-.5 z^{2}\right)$. Similarly,

$$
\begin{aligned}
\int_{-\infty}^{\underline{u}_{m}} q_{n}(u) \frac{1}{\sqrt{2 \pi}} \exp \left(-.5 u^{2}\right) d u & =-\frac{1}{\sqrt{2 \pi}} \exp \left(-.5 \underline{u}_{m}^{2}\right) \frac{1}{\underline{u}_{m}}\left(\mathcal{L}\left(\underline{q}_{n}, \underline{u}_{m}\right)+O\left(\frac{1}{\underline{u}_{m}^{3}}\right)\right) \\
=: & -L_{n}\left(\underline{u}_{m}\right)+O\left(\frac{1}{\underline{u}_{m}^{3}}\right),
\end{aligned}
$$

where $\underline{q}_{n}(z):=q_{n}\left(\underline{u}_{m}+z\right) \exp \left(-.5 z^{2}\right)$. The procedure for computing the denominator of $\tau$ in (24) is analogous.

In the general case where the quartic equation (16) has four real roots $\underline{\underline{u}}_{m} \leq \underline{u}_{m} \leq \bar{u}_{m} \leq \overline{\bar{u}}_{m}$, for instance the integral in (24) becomes

$$
\begin{aligned}
& \tau_{\text {den }}\left(y_{1}^{m-1} ; \theta_{0}\right) \\
& =\int_{-\infty}^{\underline{u}_{m}} \frac{c}{\left\|A\left(s\left(v ; \theta_{0}\right)-\tau^{(0)}\right)\right\|} d \Phi(u)+\int_{\underline{\underline{u}}_{m}}^{\underline{u}_{m}} d \Phi(u)+\int_{\underline{u}_{m}}^{\bar{u}_{m}} \frac{c}{\left\|A\left(s\left(v ; \theta_{0}\right)-\tau^{(0)}\right)\right\|} d \Phi(u) \\
& \quad+\int_{\bar{u}_{m}}^{\overline{\bar{u}}_{m}} d \Phi(u)+\int_{\overline{\bar{u}}_{m}}^{+\infty} \frac{c}{\left\|A\left(s\left(v ; \theta_{0}\right)-\tau^{(0)}\right)\right\|} d \Phi(u)
\end{aligned}
$$

and Laplace approximation could be applied to the first and the last integral. Numerical results (not reported here) show that the error when neglecting the weighting function in the central integral is very 
small. In fact $\underline{u}_{m}$ and $\bar{u}_{m}$ are typically very close and $\left\|A\left(s\left(v ; \theta_{0}\right)-\tau^{(0)}\right)\right\|$ is quite small, so that the error is essentially zero. 


\begin{tabular}{|c|c|c|c|c|c|c|c|c|}
\hline \hline & \multicolumn{2}{|c|}{$\rho_{0}=0.01$} & \multicolumn{2}{c|}{$\rho_{1}=0.8$} & \multicolumn{2}{c|}{$\alpha_{0}=0.02$} & \multicolumn{2}{c|}{$\alpha_{1}=0.8$} \\
\hline mean & 0.0100 & 0.0100 & 0.7983 & 0.7977 & 0.0200 & 0.0200 & 0.7976 & 0.8007 \\
median & 0.0099 & 0.0100 & 0.7989 & 0.7985 & 0.0199 & 0.0200 & 0.7986 & 0.8016 \\
MSE\% & 0.0026 & 0.0026 & 0.0199 & 0.0208 & 0.0002 & 0.0002 & 0.5756 & 0.5850 \\
\hline \hline mean & 0.0166 & 0.0112 & 0.7930 & 0.7959 & 0.0298 & 0.0222 & 0.8037 & 0.8081 \\
median & 0.0161 & 0.0111 & 0.7952 & 0.7965 & 0.0290 & 0.0218 & 0.8052 & 0.8064 \\
MSE\% & 0.0132 & 0.0034 & 0.0832 & 0.0254 & 0.0145 & 0.0012 & 1.7880 & 0.8097 \\
\hline \hline mean & 0.0100 & 0.0100 & 0.7982 & 0.7978 & 0.0199 & 0.0194 & 0.7989 & 0.7727 \\
median & 0.0100 & 0.0100 & 0.7988 & 0.7983 & 0.0199 & 0.0194 & 0.7992 & 0.7748 \\
MSE\% & 0.0030 & 0.0026 & 0.0256 & 0.0209 & 0.0003 & 0.0002 & 0.8006 & 0.6328 \\
\hline \hline
\end{tabular}

Table 1: Summary statistics for the PMLE (first column) and the robust estimator (second column) of the $A R(1)-A R C H(1)$ model under the reference model $\mathbb{P}_{\theta_{0}}$ (first panel; cf. also Figure 1), the replacement model (21) (second panel; cf. also Figure 2), the innovative outlier model (22) (third panel; cf. also Figure 3). 


\begin{tabular}{|c|c|c|c|c|}
\hline \hline \multirow{2}{*}{$\rho_{1}$} & \multicolumn{2}{|c|}{$t_{3}$} & \multicolumn{2}{c|}{$t_{5}$} \\
\cline { 2 - 5 } & PML & ROB & PML & ROB \\
\hline 0.00 & 0.08 & 0.05 & 0.07 & 0.05 \\
0.05 & 0.17 & 0.24 & 0.22 & 0.26 \\
0.10 & 0.46 & 0.65 & 0.62 & 0.74 \\
\hline \hline
\end{tabular}

Table 2: Each entry in the Table corresponds to the empirical rejection frequency of the joint hypothesis $\rho_{0}=0$ and $\rho_{1}=0$ obtained using $5 \%$ critical values for the $\chi^{2}$ test of the $\operatorname{AR}(1)-\operatorname{ARCH}(1)$ model under scaled $t_{3}$ and scaled $t_{5}$ innovations, respectively. 

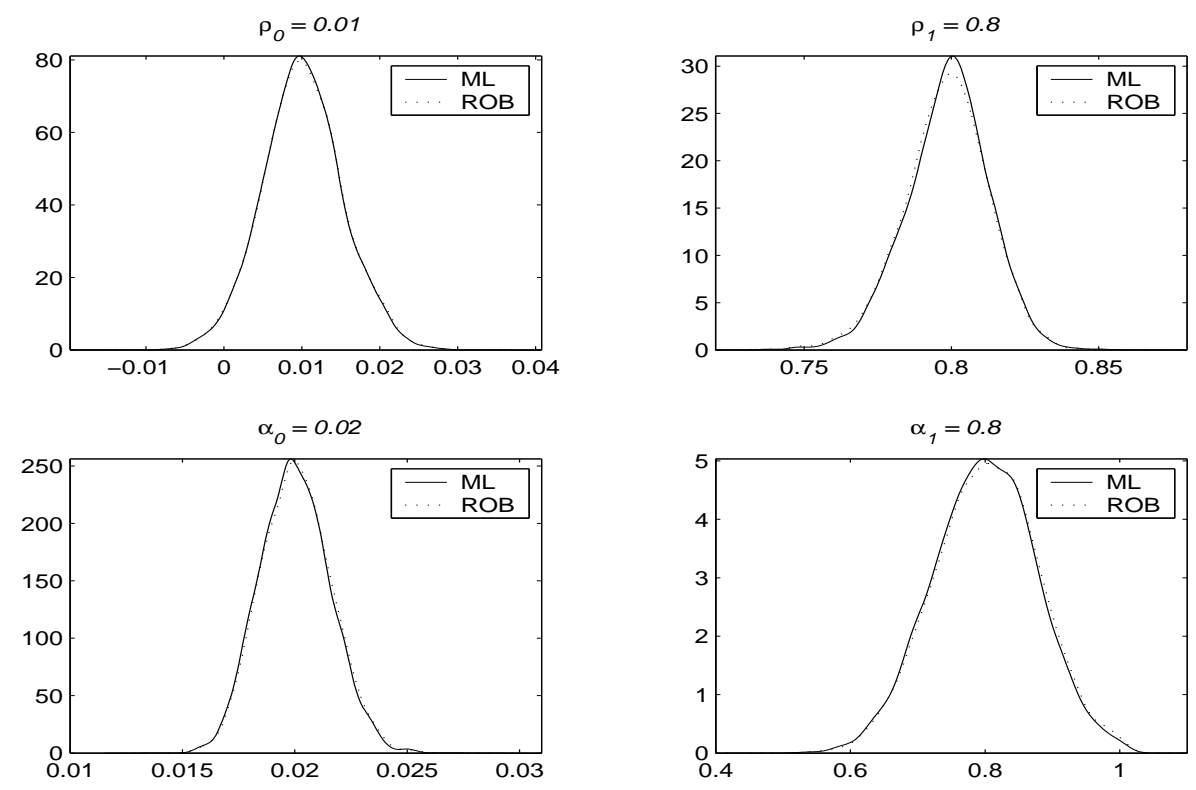

Figure 1: Estimated densities of $\hat{\theta}_{0}:=\left(\begin{array}{llll}\hat{\rho}_{0} & \hat{\rho}_{1} & \hat{\alpha}_{0} & \hat{\alpha}_{1}\end{array}\right)^{\top}$ for the $\mathrm{AR}(1)-\mathrm{ARCH}(1)$ process under the reference model $\mathbb{P}_{\theta_{0}}$, i.e. under Gaussian distribution for the innovations; cf. also the fist panel of Table 1.
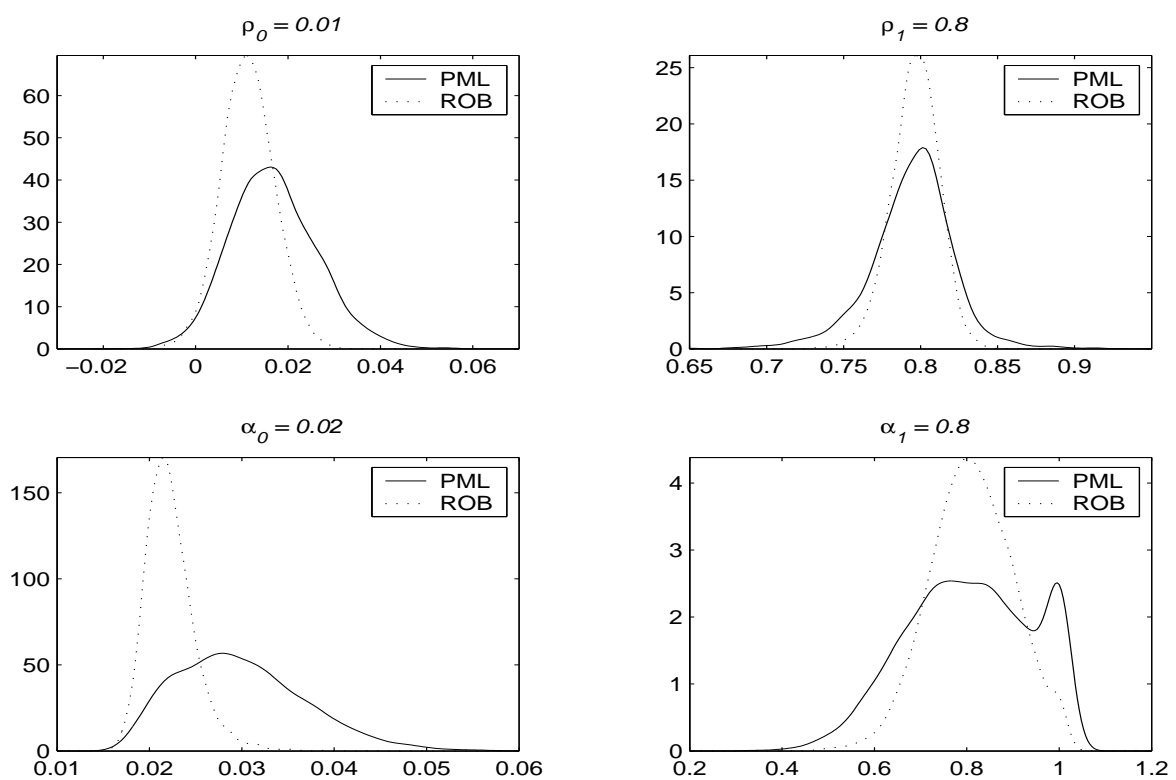

Figure 2: Estimated densities of $\hat{\theta}_{0}:=\left(\begin{array}{llll}\hat{\rho}_{0} & \hat{\rho}_{1} & \hat{\alpha}_{0} & \hat{\alpha}_{1}\end{array}\right)^{\top}$ of the $\operatorname{AR}(1)-\mathrm{ARCH}(1)$ process under the replacement model (21); cf. also the second panel of Table 1. 

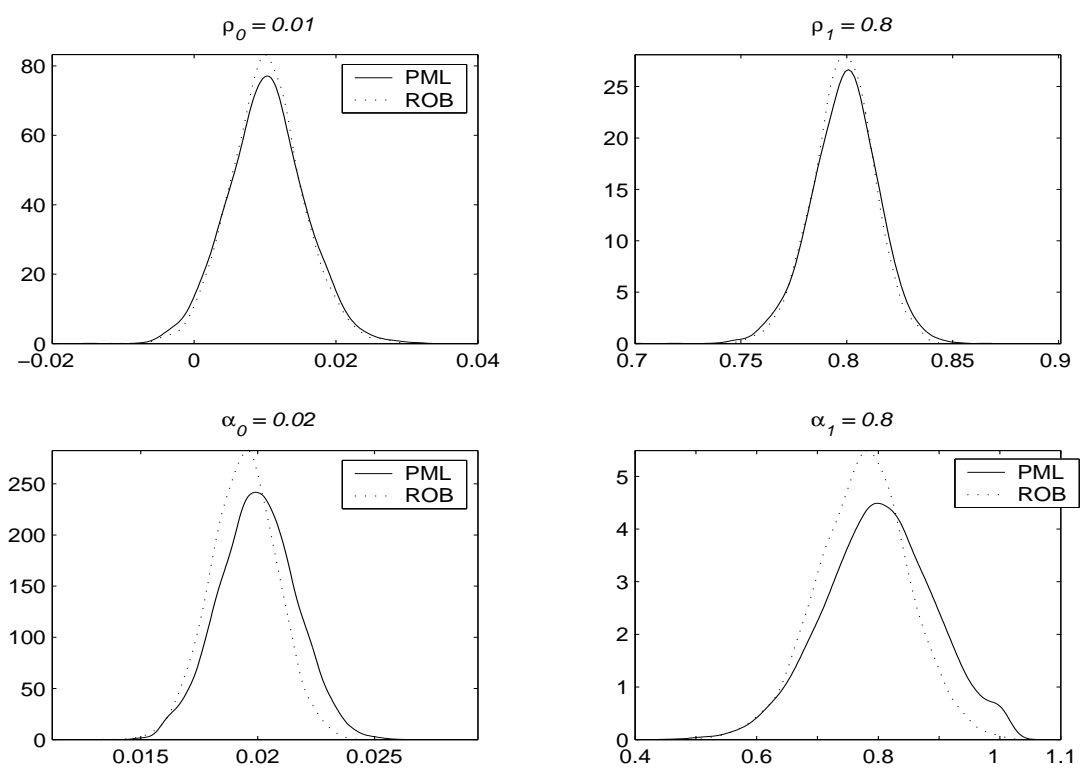

Figure 3: Estimated densities of $\hat{\theta}_{0}:=\left(\begin{array}{llll}\hat{\rho}_{0} & \hat{\rho}_{1} & \hat{\alpha}_{0} & \hat{\alpha}_{1}\end{array}\right)^{\top}$ of the $\operatorname{AR}(1)-\operatorname{ARCH}(1)$ process under the innovative outlier model (22); cf. also the third panel of Table 1.
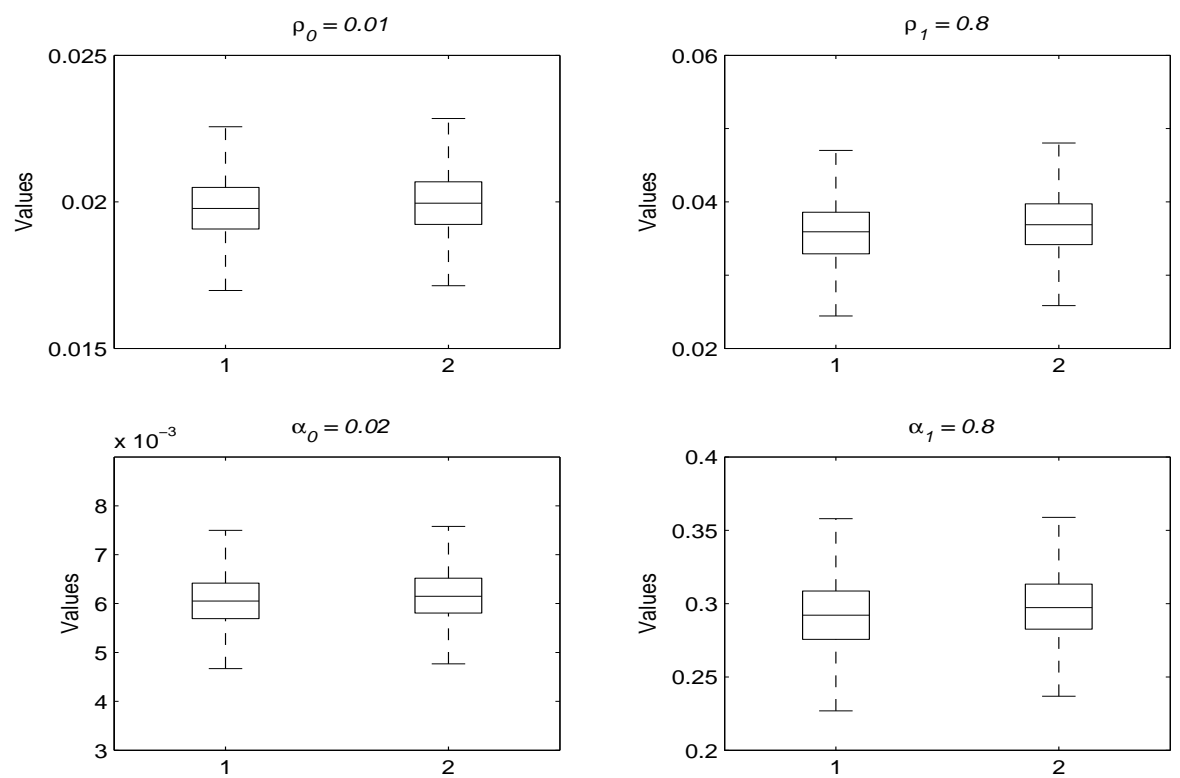

Figure 4: Boxplot of the lengths of ML (column 1) and robust (column 2) confidence intervals for $\hat{\theta}_{0}:=$ $\left(\begin{array}{llll}\hat{\rho}_{0} & \hat{\rho}_{1} & \hat{\alpha}_{0} & \hat{\alpha}_{1}\end{array}\right)^{\top}$ (cf. Figure 1) under the reference model $\mathbb{P}_{\theta_{0}}$. 

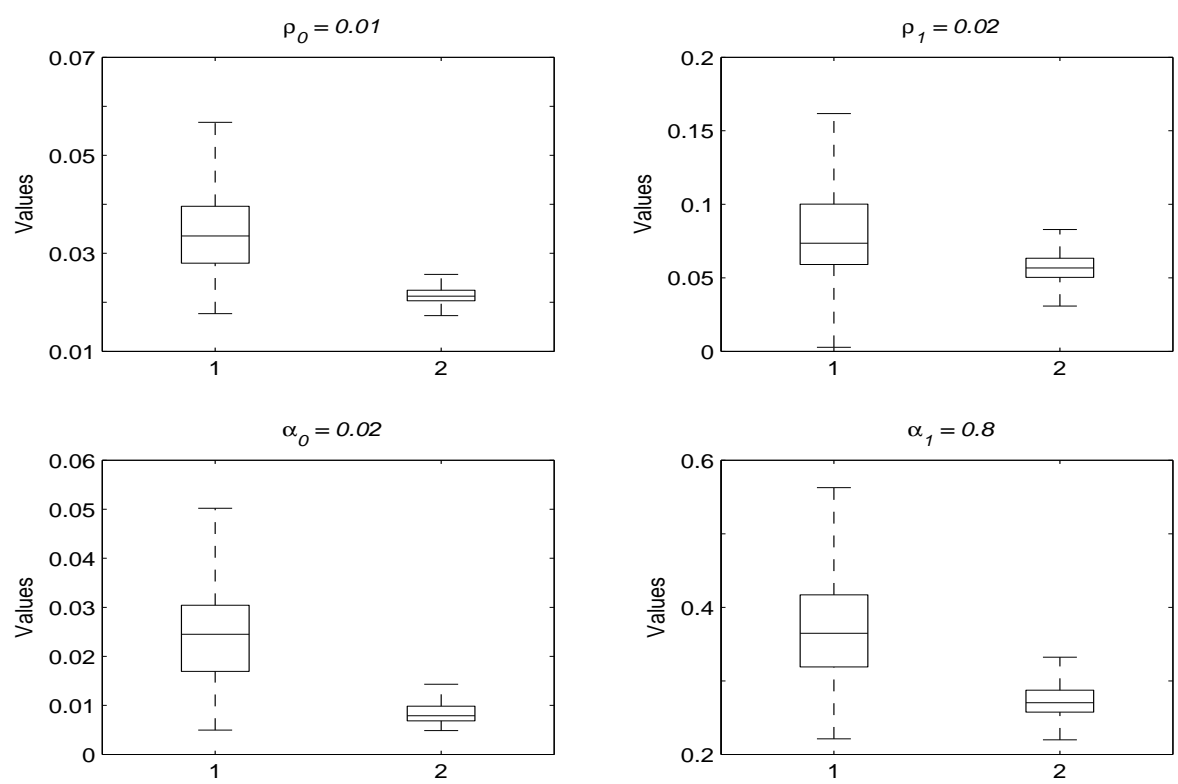

Figure 5: Boxplot of the lengths of PML (column 1) and robust (column 2) confidence intervals for $\hat{\theta}_{0}:=$ $\left(\begin{array}{llll}\hat{\rho}_{0} & \hat{\rho}_{1} & \hat{\alpha}_{0} & \hat{\alpha}_{1}\end{array}\right)^{\top}$ (cf. Figure 2) under the replacement model (21).
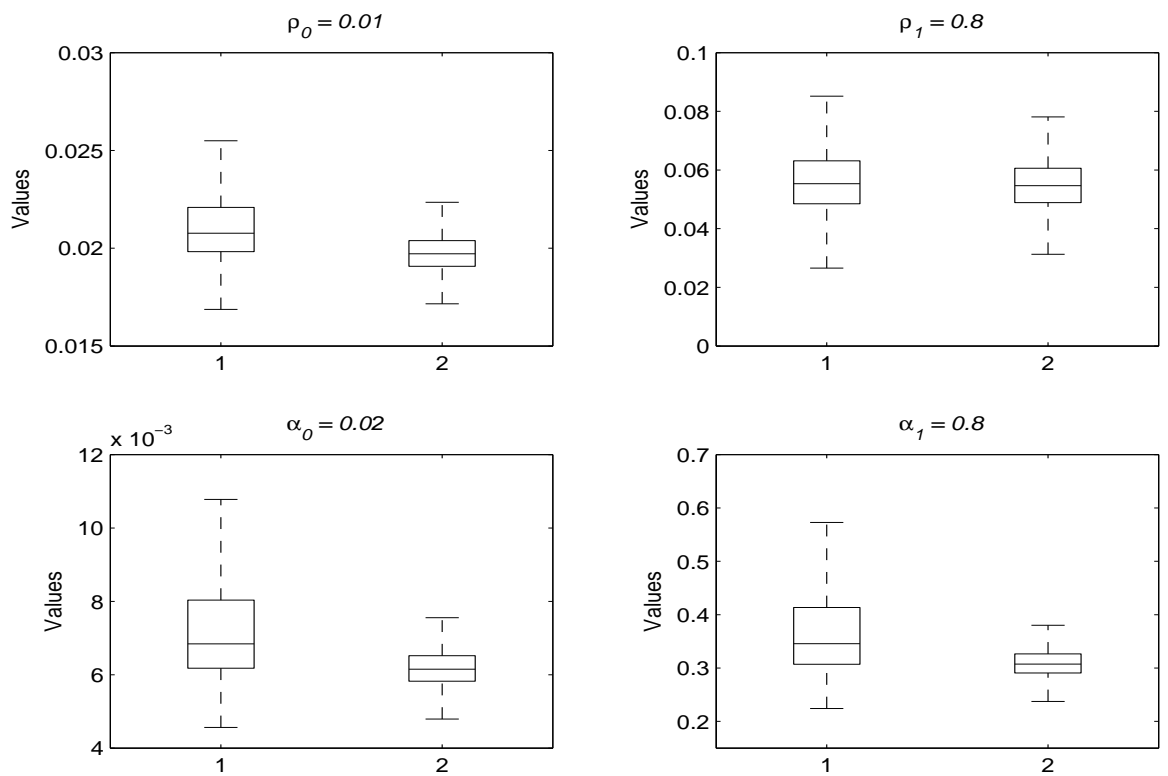

Figure 6: Boxplot of the lengths of PML (column 1) and robust (column 2) confidence intervals for $\hat{\theta}_{0}:=$ $\left(\begin{array}{llll}\hat{\rho}_{0} & \hat{\rho}_{1} & \hat{\alpha}_{0} & \hat{\alpha}_{1}\end{array}\right)^{\top}$ (cf. Figure 3) under the innovative outlier model (22). 

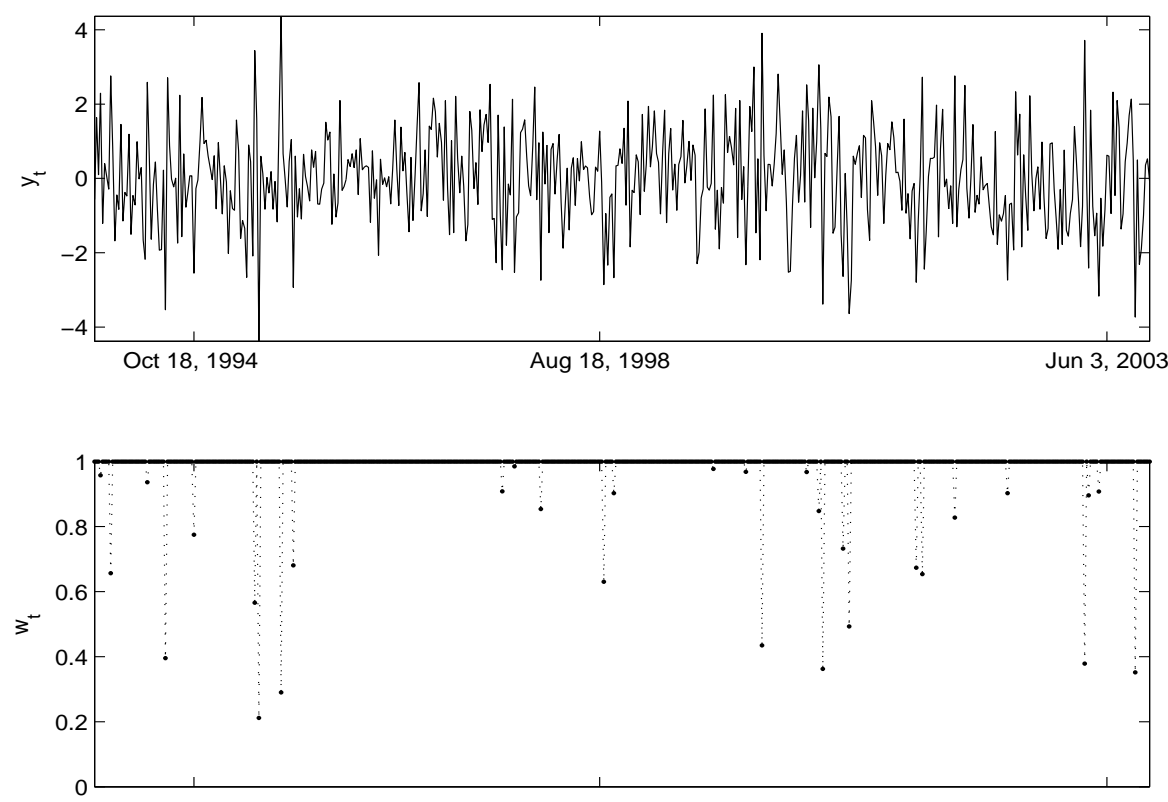

Figure 7: Weekly exchange rate returns of the Spanish peseta versus the US dollar, for the period 11/2/1993 until 10/28/2003 (top panel) and the weights implied by the robust estimate of the AR(1)-ARCH(1) model with $c=4$ (bottom panel).
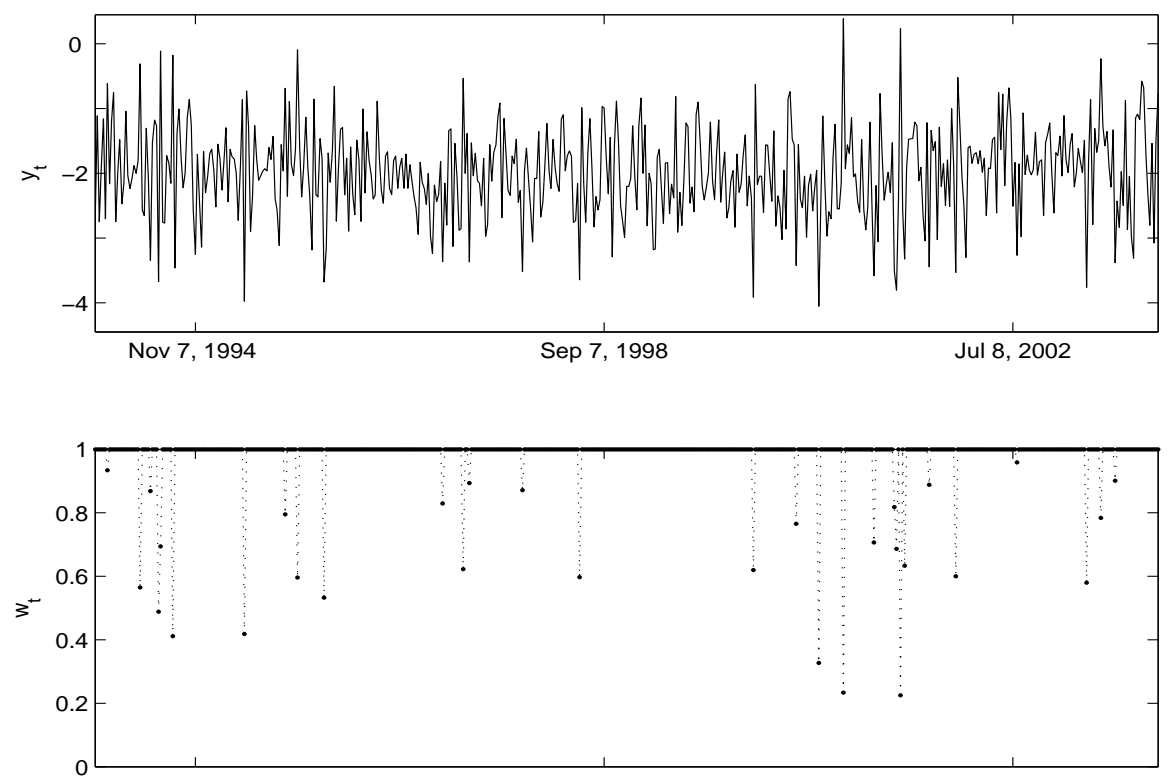

Figure 8: Weekly exchange rate returns of the Swedish krona versus the US dollar, for the period 11/29/1993 until $11 / 17 / 2003$ (top panel) and the weights implied by the robust estimate of the $A R(1)-A R C H(1)$ model with $c=4$ (bottom panel). 


\section{References}

[1] Bollerslev T.P. (1986), "Generalized Autoregressive Conditional Heteroskedasticity", Journal of Econometrics, 31, 307-327.

[2] Bollerslev T.P. And J.M. Wooldridge (1992), "Quasi-Maximum Likelihood Estimates and Inference in Dynamic Models with Time Varying Covariances", Econometric Reviews, 11, 143-172.

[3] Bustos O. And V.J. Yohai (1986), "Robust Estimates for ARMA Models", Journal of the American Statistical Association, 81, 155-168.

[4] Engle R.F. (1982), "Autoregressive Conditional Heteroskedasticity with Estimate of the Variance of United Kingdom Inflation", Econometrica, 50, 987-1007.

[5] Engle R.F., D.M. Lilien and R.P. Robins (1987), "Estimating Time Varying Risk Premia in the Term Structure: The ARCH-M Model", Econometrica, 55, 391-407.

[6] Fernholz L.T. (1983), Von Mises Calculus for Statistical Functionals, Springer, New York.

[7] Glosten L.R., R. Jagannathan and D.E. Runkle (1993), "On the Relation between the Expected Value and the Volatility of the Nominal Excess Return on Stocks", Journal of Finance, 48, $1779-1801$.

[8] Gourieroux C. and A. Monfort (1989), "A General Framework for Testing a Null Hypothesis in a Mixed Form", Econometric Theory, 5, 63-82.

[9] Gourieroux C., A. Monfort and A. Trognon (1984), "Pseudo Maximum Likelihood Methods: Theory", Econometrica, 52, 681-700.

[10] Hampel F.R. (1968), "Contributions to the Theory of Robust Estimation", Ph.D. thesis, University of California, Berkeley.

[11] Hampel F.R. (1974), "The Influence Curve and its Role in Robust Estimation", Journal of the American Statistical Association, 69, 383-393. 
[12] Hampel F.R., E.M. Ronchetti, P.J. Rousseeuw and W.A. Stahel (1986), Robust Statistics: The Approach Based on Influence Functions, Wiley, New York.

[13] Heritier S. And E. Ronchetti (1994), "Robust Bounded-Influence Tests in General Parametric Models", Journal of the American Statistical Association, 89, 897-904.

[14] Huber P.J. (1981), Robust Statistics, Wiley, New York.

[15] Jensen J.L. (1995), Saddlepoint Approximations, Clarendon Press, Oxford.

[16] Koenker R.W. (1982), "Robust Methods in Econometrics", Econometric Review, 1, 213-255.

[17] Koenker R.W. and G. Bassett (1978), "Regression Quantiles", Econometrica, 46, 33-50.

[18] Krasker W.S. and R.E. Welsh (1982), "Efficient Bounded-Influence Regression Estimation", Journal of the American Statistical Association, 77, 595-604.

[19] Krishnakumar J. And E. Ronchetti (1997), "Robust Estimators for Simultaneous Equations Models", Journal of Econometrics, 78, 295-314.

[20] KÜNsch H. (1984), "Infinitesimal Robustness for Autoregressive Processes", Annals of Statistics, 12, 843-863.

[21] Künsch H., L.A. Stefanski and R.J. Carroll (1989), "Conditionally Unbiased BoundedInfluence Estimation in General Regression Models, with Applications to Generalized Linear Models", Journal of the American Statistical Association, 84, 460-466.

[22] Li C.W. And W.K. Li (1996), "On a Double Threshold Autoregressive Heteroscedastic Time Series Model", Journal of Applied Econometrics, 11, 253-274.

[23] Markatou M. and E. Ronchetti (1997), "Robust Inference: The Approach Based on Influence Functions", in: Maddala G.S. ANd C.R. RaO (Ed.), Handbook of Statistics, vol. 15, North Holland, $49-75$.

[24] Martin R.D. and V.J. Yohai (1986), "Influence Functionals for Time Series", Annals of Statistics, 14, 781-818. 
[25] Muler N. And V.J. Yohai (1999), "Robust Estimates for ARCH Processes", Journal of Time Series Analysis, 23, 341-375.

[26] Ortelli C. and F. Trojani (2002), "Robust Efficient Method of Moments", Working Paper, University of Lugano, Switzerland.

[27] Peracchi F. (1990), "Robust M-Estimators", Econometric Review, 9, 1-30.

[28] Ronchetti E. and F. Trojani (2001), "Robust Inference with GMM Estimators", Journal of Econometrics, 101, 37-69.

[29] Sakata S. And H. White (1998), "High Breakdown Point Conditional Dispersion Estimation with Application to S\&P 500 Daily Returns Volatility", Econometrica, 66, 529-567.

[30] Stefanski L.A., R.J. Carroll and D. Ruppert (1986), "Optimally Bounded Score Functions for Generalized Linear Models with Applications to Logistic Regression", Biometrika, 73, 413-425.

[31] van Dijk D., P.H. Franses and A. Lucas (1999), "Testing for ARCH in the Presence of Additive Outliers", Journal of Applied Econometrics, 14, 539-562.

[32] von Mises R. (1947), "On the Asymptotic Distribution of Differentiable Statistical Functions", Annals of Mathematical Statistics, 18, 309-348. 